\title{
Strongly star-forming rotating disks in a complex merging system at $z=4.7$ as revealed by ALMA ${ }^{\star}$
}

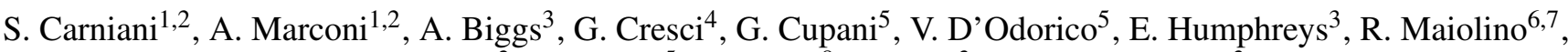

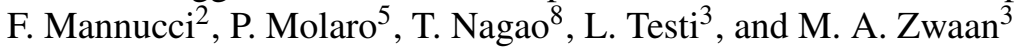 \\ ${ }^{1}$ Dipartimento di Fisica e Astronomia, Università di Firenze, via G. Sansone 1, 50019 Sesto Fiorentino (Firenze), Italy \\ e-mail: carniani@arcetri.astro.it \\ 2 INAF - Osservatorio Astrofisico di Arcetri, Largo E. Fermi 5, 50125 Firenze, Italy \\ 3 European Southern Observatory, Karl-Schwarzschild-Str. 2, 85748 Garching b. München, Germany \\ ${ }^{4}$ INAF - Osservatorio Astronomico di Bologna, Via Ranzani 1, 40127 Bologna, Italy \\ 5 INAF - Osservatorio Astronomico di Trieste, via Tiepolo 11, 34143 Trieste, Italy \\ ${ }^{6}$ Cavendish Laboratory, University of Cambridge, 19 J. J. Thomson Ave., Cambridge CB3 0HE, UK \\ 7 Kavli Institute for Cosmology, University of Cambridge, Madingley Road, Cambridge CB3 0HA, UK \\ ${ }^{8}$ Department of Astronomy, Kyoto University, Kitashirakawa-Oiwake-cho, Sakyo-ku, 606-8502 Kyoto, Japan
}

Received 18 July 2013 / Accepted 9 August 2013

\section{ABSTRACT}

\begin{abstract}
We performed a kinematical analysis of the [CII] line emission of the BR 1202-0725 system at $z \sim 4.7$ using ALMA science verification observations. The most prominent sources of this system are a quasar (QSO) and a submillimeter galaxy (SMG), separated by a projected distance of about $\sim 24 \mathrm{kpc}$ and characterized by very high star formation rates, higher than $\sim 1000 M_{\odot} \mathrm{yr}^{-1}$. However, the ALMA observations reveal that these galaxies apparently have undisturbed rotating disks, which is at variance with the commonly accepted scenario in which strong star formation activity is induced by a major merger. We also detected faint components which, after spectral deblending, were spatially resolved from the main QSO and SMG emissions. The relative velocities and positions of these components are compatible with orbital motions within the gravitational potentials generated by the QSO host galaxy and the SMG, suggesting that they are smaller galaxies in interaction or gas clouds in accretion flows of tidal streams. Moreover, we did not find any clear spectral evidence for outflows caused by active galactic nuclei or stellar feedback. This suggests that the high star formation rates might be induced by interactions or minor mergers with these companions, which do not affect the large-scale kinematics of the disks, however. Alternatively, the strong star formation may be fueled by the accretion of pristine gas from the host halo. Our kinematical analysis also indicates that the QSO and the SMG have similar dynamical masses, mostly in the form of molecular gas, and that the QSO host galaxy and the SMG are seen close to face-on with slightly different disk inclinations: the QSO host galaxy is seen almost face-on $\left(i \sim 15^{\circ}\right)$, while the SMG is seen at higher inclinations $\left(i \sim 25^{\circ}\right)$. Finally, the ratio between the black hole mass of the QSO, obtained from new X-shooter spectroscopy, and the dynamical mass of the host galaxy is similar to value found in very massive local galaxies, suggesting that the evolution of black hole galaxy relations is probably better studied with dynamical than with stellar host galaxy masses.
\end{abstract}

Key words. galaxies: kinematics and dynamics - galaxies: evolution - galaxies: high-redshift - quasars: general galaxies: starburst - galaxies: star formation

\section{Introduction}

The growth of galaxies is closely traced by star formation, and the cosmological evolution of the global star formation rate (SFR) density is thus a fundamental element for understanding galaxy evolution from high to low redshift. The cosmic SFR density peaks at about $z=1 \sim 3$, as illustrated by the so-called Madau-Lilly diagram (Lilly et al. 1996; Madau et al. 1996; Haarsma et al. 2000; Hopkins et al. 2001). Until recently, galaxy evolutionary models usually associated this peak with the increased rate of galaxy mergers and interactions in that redshift range. In particular, the merger process is at the base of the formation of massive galaxies: the majority of the stars are formed following major-merger episodes that induce high SFRs (from several hundreds to over a thousand $M_{\odot} \mathrm{yr}^{-1}$; Di Matteo et al. 2005; Guyon et al. 2006; Sijacki et al. 2011; Valiante et al. 2011).

\footnotetext{
* Reduced datacubes (FITS files) are only available at the CDS via anonymous ftp to cdsarc.u-strasbg. fr (130.79.128.5) or via http://cdsarc.u-strasbg.fr/viz-bin/qcat?J/A+A/559/A29
}

But a different point of view has emerged very recently, according to which the peak of the cosmic SFR density is expected to follow the evolution of the cosmic gas inflow rate. This is suggested by the existence of a main-sequence of star-forming galaxies at any redshift, which is most likely fueled by continuous gas accretion (Kereš et al. 2005; Genel et al. 2008; Conselice et al. 2013).

Strongly starbursting outliers of such a main sequence do exist and represent an increasingly important phase at higher redshifts: these outliers are thought to be mostly merger-induced star-forming objects, such as submillimeter and ultraluminous infrared galaxies (Tacconi et al. 2010), even if some authors have found that the fraction of merger systems is small (Rodighiero et al. 2010). Whatever the origin of the evolution of the cosmic SFR density, submillimeter galaxies (SMGs) appear to fit well within a merger-induced star formation scenario. They are dustobscured strongly star-forming galaxies whose number density peaks around $z \sim 2$ (Chapman et al. 2003, 2005), although recent ALMA surveys have shown that the SMG redshift distribution 
probably peaks at even higher redshift $(z \sim 3.5$, Weiß et al. 2013). Their luminosities are in excess of $10^{12} L_{\odot}$, their SFRs are of about $\sim 10^{2}-10^{3} M_{\odot} \mathrm{yr}^{-1}$, and their stellar masses are around $10^{11} M_{\odot}$, although recent estimates made by different authors vary by a factor $\sim 6$ (Magnelli et al. 2010; Chapman et al. 2010; Hainline et al. 2011; Michałowski et al. 2012; Magnelli et al. 2012). When combining these properties with typical gas fractions of $40 \%$ (Narayanan et al. 2012), it is natural to assume that SMGs are the late stages of major-merger events.

However, in contrast with the major-merger scenario, a large portion of the strongly star-forming galaxies at $z \sim 1-3$, especially among Lyman-break galaxies, does not show the expected disturbed kinematics but is characterized by regularly rotating disks (Förster Schreiber et al. 2009; Epinat et al. 2009; Cresci et al. 2009; Gnerucci et al. 2011a). This observational result has prompted the suggestion that even stronger star formation may be fueled by the accretion of pristine gas from the halo and dynamical instabilities within the massive gaseous disks (Genel et al. 2008; Dekel et al. 2009; Bouché et al. 2010; Cresci et al. 2010). Another evidence of this smooth accretion is provided by the so-called fundamental metallicity relation, a tight relation for star-forming galaxies that connects gas metallicity, SFR and stellar mass, and which does not evolve with redshift up to $z \sim 3$ (Mannucci et al. 2010; Dayal et al. 2013; Bothwell et al. 2013).

Star formation is not the only ingredient in the formation of galaxies. The discovery of the correlations between the masses of supermassive black holes and the properties of their host spheroids (Ferrarese \& Merritt 2000; Gebhardt et al. 2000; Marconi \& Hunt 2003; Gültekin et al. 2009; McConnell \& Ma 2013) and the observed cosmological evolution of the active galactic nuclei (AGN) number density, which follows that of the cosmic SFR density (Marconi et al. 2004; Merloni \& Heinz 2008; Shankar et al. 2010), have suggested that the growth of black holes (BHs) is intimately linked with that of their host galaxies: this so-called co-evolution is also based on the merger paradigm (e.g. Hopkins et al. 2007). In particular, in the most luminous AGN, major-merger episodes destabilize large amounts of gas, which forms stars and eventually accretes onto the central supermassive black holes. The luminous AGN that are then activated have feedback onto their host galaxies: fast radiation-driven outflows start sweeping away the surrounding gas, quenching both black hole accretion and star formation. This is the beginning the observed black hole galaxy correlations and explains the decrease of the cosmic SFR and AGN densities below $z \sim 1$. The co-evolution scenario then implies that there should be a population of active, clustered star-forming galaxies at high redshift $(z>3)$.

Observations of the morphology and kinematics of molecular gas in high-redshift galaxies may help to understand the main mechanism that caused the peak of the cosmic SFR density. In particular, the kinematics of gas in merging systems might indicate whether star formation activity is indeed associated with disturbed kinematics. Moreover, the distribution of ionized and molecular gas in active high-redshift sources such as quasars might reveal outflows, as expected from the feedback model.

BR 1202-0725 is an ideal system to test these scenarios as it is composed of strongly star forming interacting galaxies at redshift $z \sim 4.7$. The most prominent sources are a quasar (QSO) at the southeast and a SMG at the northwest. The bright QSO $\left(M_{\mathrm{B}}=-28.5\right)$ presents broad Ly $\alpha$ emission extended toward the northwest, which suggests interaction with the SMG (Hu et al. 1996). The SMG has been observed for the first time at $1.4 \mathrm{GHz}$ (Carilli et al. 2002) and is located about $24 \mathrm{kpc}$ from the QSO (Wagg et al. 2012). The observed flux level at
$850 \mu \mathrm{m}$ (Iono et al. 2006) places the SMG at the bright end of the SMG population $(S[850 \mu \mathrm{m}]>5)$, which represents only $20-30 \%$ of the submm sources responsible for the $850 \mu \mathrm{m}$ extragalactic background radiation (e.g. Coppin et al. 2006). Both sources have a high far-infrared (FIR, 40-500 $\mu \mathrm{m}$ ) luminosity, $L_{\text {FIR }}>10^{13} L_{\odot}$ (Iono et al. 2006; Omont et al. 1996; Carilli et al. 2002) that likely indicates very strong star formation activity $\left(S F R>10^{3} M_{\odot} \mathrm{yr}^{-1}\right)$. Assuming that warm dust is heated by star formation activity and re-emits in the FIR, it is possible to derive an $S F R \sim 4500 M_{\odot} \mathrm{yr}^{-1}$ for the QSO and an $S F R \sim 2000 M_{\odot} \mathrm{yr}^{-1}$ for the SMG (Iono et al. 2006).

The system BR 1202-0725 has been recently observed with ALMA during the science demonstration phase (Wagg et al. 2012; Carilli et al. 2013) and with the IRAM PdB Interferometer as reported by Salomé et al. (2012). These authors derived SFRs of about $1000 M_{\odot} \mathrm{yr}^{-1}$ and molecular gas masses of $\sim 5 \times$ $10^{10} M_{\odot}$. Moreover, the system has been found to be more complex than previously thought. Wagg et al. (2012) and Carilli et al. (2013) detected two fainter sources, one southwest of the quasar and one in between the QSO and the SMG, which are the [CII] counterparts of known Ly $\alpha$ emitters; furthermore, the $\mathrm{CO}$ line profile and position-velocity diagram by Salomé et al. (2012) indicate a close companion also for the SMG. Intriguingly, the CO position-velocity diagrams for both the QSO and the SMG suggest that the two galaxies have regularly rotating disks.

Here we perform a kinematical analysis of the [CII] line emission in BR 1202-0725 from the observations obtained with ALMA during the science demonstration phase. We show that, at variance with a simple major-merger scenario, the high SFRs in both the QSO host galaxy and the SMG are associated with apparently regularly rotating disks, suggesting that the high SFRs are probably induced by minor mergers, interactions, or gas inflows that do not strongly affect the large-scale disk kinematics. We determine the dynamical masses of the two galaxies and study the relation between black hole and host galaxy for the QSO. Data reduction is described in Sect. 2 and results from the data analysis are presented in Sect. 3. In Sect. 4 we present our kinematical analysis and the dynamical mass measurements of the QSO host galaxy and SMG. We discuss our findings in Sect. 6 and finally draw our conclusions in Sect. 7.

Throughout this paper we adopt the standard cosmological parameters $H_{0}=70 \mathrm{~km} \mathrm{~s}^{-1} \mathrm{Mpc}^{-1}, \Omega_{\mathrm{M}}=0.30$ and $\Omega_{\Lambda}=0.70$, according to which $1 \operatorname{arcsec}$ at $z=4.7$ corresponds to a proper distance of $6.48 \mathrm{kpc}$.

\section{Observations and data reduction}

BR 1202-0725 was observed at $0.9 \mathrm{~mm}$ (334 GHz) using the Atacama Large Millimeter/Submillimeter Array, ALMA, with 18 12-m and 3 7-m diameter antennas on 2012 January 16; for this work we took advantage of all the available data. The total on-source observing time was about $25 \mathrm{~min}$ with a maximum baseline length $\sim 280 \mathrm{~m}$. The weather conditions were generally very good with a precipitable water vapor of $0.7 \mathrm{~mm}$, and the continuum sensitivity of ALMA was one order of magnitude higher than previous submillimeter observations of the system.

The receivers were tuned to a [CII] redshifted frequency of about $333 \mathrm{GHz}$ (the rest frequency of [CII] is $1900.539 \mathrm{GHz}$ ). The ALMA correlator has a total bandwidth of $7.5 \mathrm{GHz}$ and was divided in to four spectral windows of $\sim 1.8 \mathrm{GHz}$ with a channel width of $15.6 \mathrm{MHz}\left(\sim 14 \mathrm{~km} \mathrm{~s}^{-1}\right)$. The data were calibrated by using the CASA software. The amplitude and phase fluctuations were calibrated by observations of $3 \mathrm{C} 279$, for which we took the flux of $15 \mathrm{Jy}$ obtained from SMA observation at $850 \mu \mathrm{m}$. 
Table 1. Summary of the physical properties of the BR 1202-0725 system from the literature and from this paper.

\begin{tabular}{|c|c|c|c|}
\hline & \multicolumn{2}{|c|}{ Sources } & \multirow[b]{2}{*}{ References } \\
\hline & BR $1202 \mathrm{~N}$ & BR $1202 \mathrm{~S}$ & \\
\hline RA & 12:05:22.98 & $12: 05: 23.13$ & \\
\hline Dec & $-07.42 .29,56$ & $-07.42 .32,68$ & \\
\hline Integrated continuum flux at $900 \mu \mathrm{m}[\mathrm{mJy}]$ & $15.8 \pm 2.4$ & $14.8 \pm 2.2$ & \\
\hline Distance from S source $[\mathrm{kpc}]$ & $23.5 \pm 0.3$ & - & \\
\hline FWHM from Gaussian fitting of continuum & $0.84^{\prime \prime} \times 0.75^{\prime \prime}$ & $0.81^{\prime \prime} \times 0.78^{\prime \prime}$ & \\
\hline Integrated $[\mathrm{CII}]$ flux $\left[\mathrm{Jy} / \mathrm{km} \mathrm{s}^{-1}\right]$ & 9.06 & 8.45 & \\
\hline FHWM of $[\mathrm{CII}]$ line $\left[\mathrm{km} \mathrm{s}^{-1}\right]$ & $500 \pm 20$ & $300 \pm 20$ & \\
\hline Redshift & 4.6891 & 4.6943 & \\
\hline$L_{\mathrm{FIR}}\left[L_{\odot}\right]$ & $1.5 \times 10^{13}$ & $1.8 \times 10^{13}$ & \\
\hline SFR from continuum $\left[M_{\odot} \mathrm{yr}^{-1}\right]$ & 2600 & 3200 & \\
\hline SFR from $[\mathrm{CII}]\left[M_{\odot} \mathrm{yr}^{-1}\right]$ & $6800 \pm 1000$ & $5000 \pm 1000$ & \\
\hline$L_{\mathrm{CO}}^{\prime}\left[10^{10} \mathrm{~K} \mathrm{~km} \mathrm{~s}^{-1} \mathrm{pc}^{2}\right]$ & $7.8 \pm 1.2$ & $5.3 \pm 0.8$ & 1 \\
\hline$\alpha_{\mathrm{CO}}$ & $0.6 \pm 0.2$ & $0.6 \pm 0.2$ & 2 \\
\hline Molecular mass $\left[10^{10} M_{\odot}\right]$ & $4.6 \pm 1.7$ & $3.2 \pm 1.2$ & \\
\hline Disk inclination $\left[{ }^{\circ}\right]$ & $25 \pm 15$ & $15 \pm 10$ & \\
\hline PA of line of nodes $\left[{ }^{\circ}\right]$ & $180 \pm 5$ & $230 \pm 10$ & \\
\hline Scale radius $[\mathrm{kpc}]$ & $2.8 \pm 0.2$ & $2.4 \pm 0.2$ & \\
\hline $\log _{10}\left(M_{\mathrm{dyn}} / M_{\odot}\right)$ & $10.8 \pm 0.6$ & $10.6_{-0.4}^{+0.8}$ & \\
\hline $\log _{10}\left(M_{\mathrm{BH}} / M_{\odot}\right)$ & - & $9.1 \pm 0.3$ & \\
\hline$M_{\mathrm{BH}}\left[10^{9} M_{\odot}\right]$ & - & $1.5_{-0.8}^{+1.5}$ & \\
\hline$\Sigma_{\mathrm{SFR}}\left[M_{\odot} / \mathrm{yr} \mathrm{kpc}^{-2}\right]$ & 80 & 180 & \\
\hline$\Sigma_{\mathrm{gas}}\left[M_{\odot} \mathrm{pc}^{-2}\right]$ & 1900 & 1800 & \\
\hline Dark halo mass $\left[M_{\odot}\right]$ & \multicolumn{2}{|c|}{$>3.2 \times 10^{11}$} & \\
\hline
\end{tabular}

References. (1) Salomé et al. (2012) ; (2) Bolatto et al. (2013).

Afterwards we used observations of Titan to rescale the flux, assuming a total value of $2.5 \mathrm{Jy}$. The quasar 3C 279 was also used to calibrate the time-dependent gain and the bandpass. Overall, we estimate a $15 \%$ uncertainty on the measured absolute fluxes.

The high sensitivity of ALMA has allowed a self-calibration of the BR 1202-0725 data using the line-free channels: we used a 3-min averaging time for the complex gain solutions in the amplitude and phase self-calibration.

The continuum image was extracted using all the line-free channels in the last three spectral windows; the image was then cleaned using the CASA task "CLEAN" with ROBUST $=1.5$, achieving an angular resolution of $0.8^{\prime \prime} \times 0.7^{\prime \prime}$ with the beam oriented at $\mathrm{PA}=8^{\circ}$. The achieved sensitivity in the continuum image is $0.2 \mathrm{mJy}$ (rms/beam).

A UV plane model of the continuum emission was made from the line-free channels of the last three spectral windows and was subtracted from the first and second spectral windows after linear extrapolation. Following the same deconvolution procedure as for the continuum image, we then obtained the final line data cube with the first two spectral windows rebinned to channels of $\sim 28 \mathrm{~km} \mathrm{~s}^{-1}$. The spatial scale is $0.15^{\prime \prime}$ per pixel $(\sim 0.97 \mathrm{kpc}$ per pixel). The rms noise was estimated to be $2.0 \mathrm{mJy}$ per beam per channel, from several line-free channels.

The continuum and line maps obtained by us are similar to those provided with the data release. Continuum and line fluxes are consistent within the errors and the main difference consists in our choice of pixel scale and ROBUST values, which improved the spatial resolution by a factor $\sim 2$.

\section{Results}

Table 1 summarizes the physical properties of the BR 1202-0275 system as measured in this paper or collected from the literature. Here we identified the two brightest sources with the labels $\mathrm{N}$

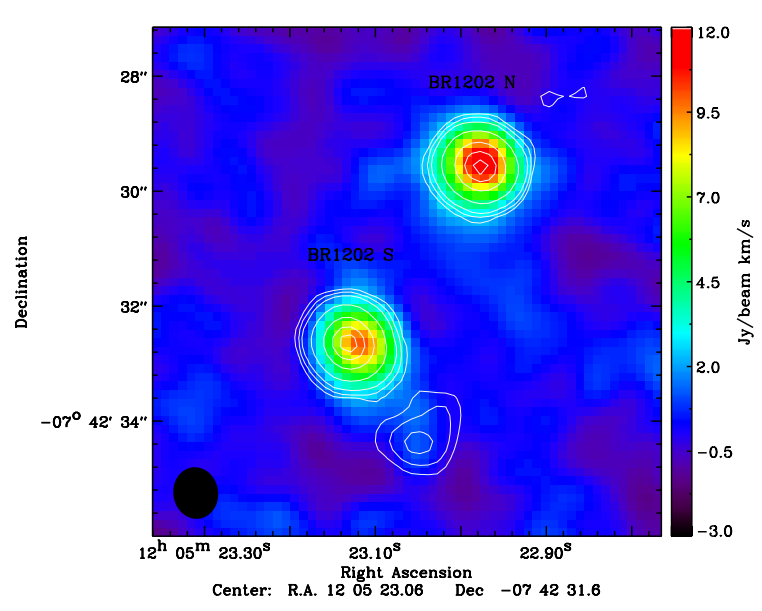

Fig. 1. [CII] integrated intensity map in BR 1202-0725 with identification of the north and south sources. The rms noise is $0.6 \mathrm{Jy} \mathrm{km} \mathrm{s}^{-1}$. The synthesized beam $\left(0.8^{\prime \prime} \times 0.7^{\prime \prime}\right)$ is indicated by a filled black ellipse in the lower left corner of the plot. The continuum emission of BR 12020725 a $334 \mathrm{GHz}$ is overplotted as white contours. The contour levels are logarithmic $[-3.4,-3.2,-3.0,-2.6,2.2,2.0,1.9]$ of $\sigma$ with $\sigma=0.2$ mJy. The rms noise is $0.2 \mathrm{mJy}$ beam $^{-1}$.

and S (north and south). The north source is the SMG and the south source is the QSO.

Figure 1 presents the [CII] intensity map of the BR 12020275 system with the surface brightness contours of the $900 \mu \mathrm{m}$ continuum emission overlaid. The integrated fluxes of the $\mathrm{N}$ and $\mathrm{S}$ sources at $334 \mathrm{GHz}$ and in the [CII] line and their centroid coordinates (Table 1) are consistent with those found by Iono et al. (2006) and the previous analysis of these data by Wagg et al. (2012) and Carilli et al. (2013). The projected distance between the two continuum sources is $23.5 \pm 0.3 \mathrm{kpc}$. The fainter continuum source discovered by Wagg et al. (2012) southwest of 

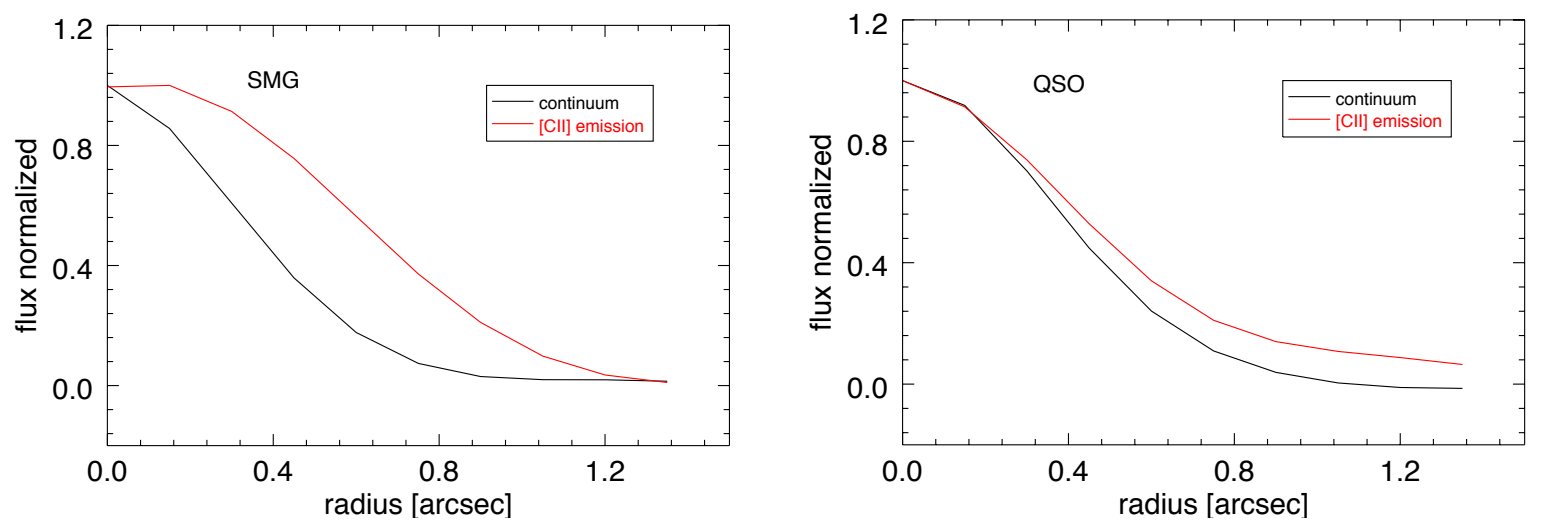

Fig. 2. Radial surface brightness profiles of the SMG (N source, left panel) and of the quasar (S source, right panel). The continuum and [CII] line emissions are denoted by the black and red lines, respectively.
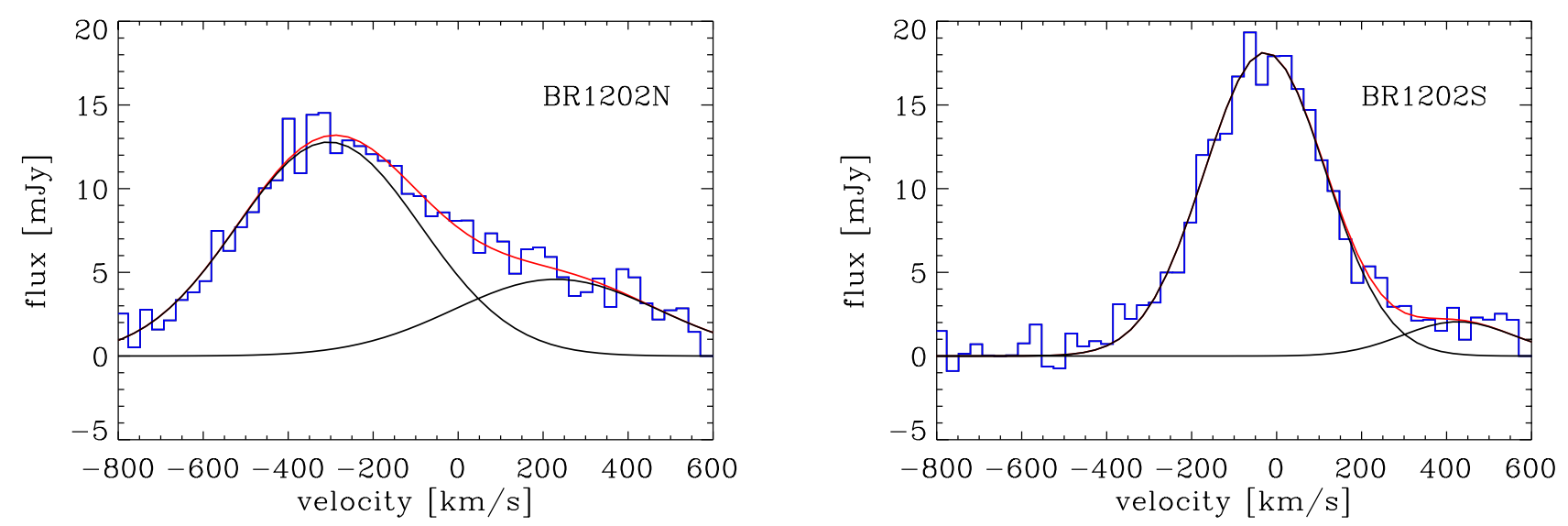

Fig. 3. Integrated spectra of the $\mathrm{N}$ and $\mathrm{S}$ sources (left and right panels, respectively), targeting the [CII] line with a spectral resolution of $28 \mathrm{~km} \mathrm{~s}{ }^{-1}$ per channel. The spectra have been extracted from the ALMA data cubes using beam-sized apertures centered on the sources. All velocities are referred to a redshift $z=4.6949$. The red curves denote the best fits, which are obtained with a double Gaussian function for both sources. For the QSO the width of the second component is fixed to that of the main component. For the SMG, the velocity of the fainter component is fixed at $250 \mathrm{~km} \mathrm{~s}^{-1}$ (Salomé et al. 2012).

the QSO (Fig. 1) is detected in the continuum at the $\sim 8 \sigma$ level $\left(\sigma=0.2\right.$ mJy beam $\left.^{-1}\right)$. This source is not observed in the integrated line map of Fig. 1 because of the large velocity range used in integration.

The continuum emission of the N and S sources is not spatially resolved because their FWHM are similar, within the errors, to that of the synthesized beam (Table 1). The line emission of both sources, however, is spatially resolved compared to the continuum, as shown by the FWHM in Table 1 and by the radial surface brightness profiles presented in Fig. 2. As shown in the figure for both the $\mathrm{N}$ and $\mathrm{S}$ sources, the radially averaged FWHM of the [CII] emission along the $x$-axis $(\mathrm{E}-\mathrm{W})$ is $\sim 1.2$ times higher than that of the synthesized beam and of the continuum. Moreover, it is clear from Fig. 1 that the peak of [CII] emission in the QSO (S source) is shifted by $0.2^{\prime \prime}(\sim 1.3 \mathrm{kpc})$ with respect to the continuum.

The integrated spectra of the $\mathrm{N}$ and $\mathrm{S}$ sources are shown in Fig. 3. The line profile of the QSO is generally symmetric, except for a "red wing" at $\sim 500 \mathrm{~km} \mathrm{~s}^{-1}$ that is probably associated with a faint companion or with an outflowing wind, as suggested by Carilli et al. (2013). To take into account the red wing, the spectrum was fit with two Gaussian functions with the same FWHM because the red wing is at the edge of the band and not fully constrainable. As discussed below, this choice does not affect the kinematics of the QSO host galaxy. Conversely, the line profile of the SMG is asymmetric and broader than that of the quasar. It was fit with a combination of two Gaussians with the fainter component fixed at $250 \mathrm{~km} \mathrm{~s}^{-1}$, as suggested by Salomé et al. (2012) and explained in more detail below. The results, including average velocities and velocity dispersions, are presented in Table 1. The redshifts of the two sources are determined from a Gaussian fit to the [CII] lines and corresponds to $z=4.6891 \pm 0.0004$ for the SMG and $z=4.6943 \pm 0.0005$ for the QSO. In all cases [CII] velocities are referred to the systemic redshift of $z=4.6949$ adopted by Salomé et al. (2012); our velocity and the velocity dispersion measurements for [CII] are consistent with the measurements by Carilli et al. (2013), Wagg et al. (2012), Iono et al. (2006), and with the corresponding CO values by Salomé et al. (2012).

\subsection{Star formation rate and gas mass}

We first estimated the SFR from the FIR continuum emission. To this aim, we fit the spectral energy distributions (SEDs) of the two sources in the FIR-radio range using the model of Yun \& Carilli (2002) and assuming typical values for the dust temperatures $\left(T_{\mathrm{d}}\right)$ and opacities $\left(k_{v}\right)$ of SMGs and quasars $\left(T_{\mathrm{d}}=65 \mathrm{~K}\right.$ for the SMG and $T_{\mathrm{d}}=50 \mathrm{~K}$ for the QSO; $k_{v} \sim v^{1.5}$; see Iono et al. 2006; Beelen et al. 2006; Kovács et al. 2006, for more details). Fluxes for BR 1202N and BR 1202S were compiled from 

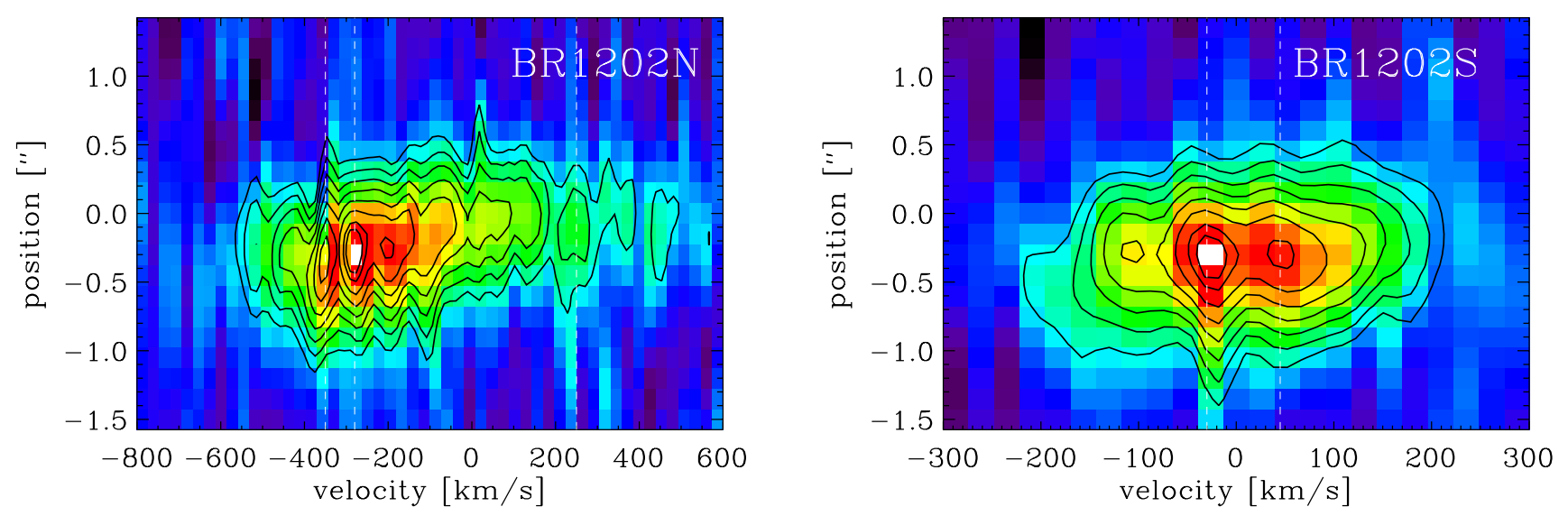

Fig. 4. Left panel: the position-velocity diagram extracted from a three-pixel-wide E-W slit centered on the N source (SMG). The right panel is the PV diagram for a slit oriented N-S and centered on the S source (QSO). The white dashed lines identify the components discussed in the text.

Carilli et al. (2002, $1.4 \mathrm{GHz})$, Omont et al. (1996, $4.8 \mathrm{GHz})$, Benford et al. $(1999,1000 \mathrm{GHz})$ and from our own ALMA measurement $(334 \mathrm{GHz})$. The adopted SED model is composed of thermal bremsstrahlung, nonthermal synchrotron and dust continuum emission; the latter depends on the SFR, which is the only free parameter of our SED fit. From this we estimate a SFR of $\sim 4000 M_{\odot} \mathrm{yr}^{-1}$ for the $\mathrm{N}$ galaxy and $\sim 5000 M_{\odot} \mathrm{yr}^{-1}$ for the S source.

Using the SED fitting only for estimating the FIR luminosity and applying the well-known SFR-FIR relation

$S F R=1.73 \times 10^{-10}\left(L_{\mathrm{FIR}} / L_{\odot}\right) M_{\odot} / \mathrm{yr}$

by Kennicutt (1998), one obtains $\sim 2600$ and $\sim 3200 M_{\odot} \mathrm{yr}^{-1}$ for the SMG and QSO, respectively. These values are similar to the previous ones and to those found by Iono et al. (2006) with the same relation. The adopted SFR-FIR relation was calibrated with a Salpeter IMF; the use of a top-heavy Kroupa IMF would lower the SFRs by a factor $\sim 1.6$ (see Salomé et al. 2012; and Mor et al. 2012, for more details).

The SFR can also be estimated from the luminosity of the [CII] emission line, since it has been shown to be a tracer of star formation activity in different types of galaxies (Genzel \& Cesarsky 2000; Malhotra et al. 2001; Luhman et al. 2003). Using the calibration of Maiolino et al. (2005), we estimate an SFR of $\sim 6800 M_{\odot} \mathrm{yr}^{-1}$ for the SMG and of $\sim 5000 M_{\odot} \mathrm{yr}^{-1}$ for the QSO. The calibration of Sargsyan et al. (2012) would provide much lower SFRs of less than $900 M_{\odot} \mathrm{yr}^{-1}$ for both sources, but this calibration may not be applicable since it is obtained from galaxies at lower redshifts $(z \sim 1-2)$ and with lower star formation rates $\left(S F R<500 M_{\odot} \mathrm{yr}^{-1}\right)$. Given the uncertainties on the use of [CII] as an SFR indicator and the possibility that [CII] could trace the cold neutral interstellar medium, in the following we used only SFRs based on the FIR luminosity and considered the values based on [CII] just as a confirmation of the very high SFR.

We estimated the molecular gas masses from $L_{\mathrm{CO}}^{\prime}(5-4)$ (Salomé et al. 2012) using the conversion factor $\alpha_{\mathrm{CO}}=0.6 \pm$ $0.2 M_{\odot}\left(\mathrm{K} \mathrm{km} \mathrm{s}^{-1} \mathrm{pc}^{2}\right)^{-1}$ resulting from one-component modeling in a large sample of starburst galaxies (Papadopoulos et al. 2012). For comparison, Salomé et al. (2012) used the classical value of $\alpha_{\mathrm{CO}}=0.8 . M_{\odot}\left(\mathrm{K} \mathrm{km} \mathrm{s}^{-1} \mathrm{pc}^{2}\right)^{-1}$ from Downes \& Solomon (1998). As discussed in detail by Bolatto et al. (2013), the $\alpha_{\mathrm{CO}}$ conversion factor is still very uncertain especially at high redshift and, for this reason, we adopted a factor 2 ( $0.3 \mathrm{dex})$ systematic uncertainty on $\alpha_{\mathrm{CO}}$. The molecular masses are therefore
$M_{\mathrm{CO}}=(4.6 \pm 1.7) \times 10^{10} M_{\odot}$ and $M_{\mathrm{CO}}=(3.2 \pm 1.2) \times 10^{10} M_{\odot}$ for the SMG and the QSO host galaxy, respectively. These measurements are also affected by the factor 2 systematic uncertainty on $\alpha_{\mathrm{CO}}$.

Summarizing, the FIR and [CII] based indicators provide SFRs between $\sim 1000-5000 M_{\odot} \mathrm{yr}^{-1}$ for the two sources, which are associated with very large masses of molecular gas $\left(M_{\mathrm{mol}} \sim\right.$ $\left.4 \times 10^{10} M_{\odot}\right)$. Overall, these results suggest extreme star formation activity, like that associated with major-merger events, and place the two sources at the higher end of the SFR distribution for star-forming galaxies. To verify this scenario and determine whether the two galaxies show the disturbed kinematics typical of a major merging event, we performed a detailed kinematical analysis.

\section{Kinematical analysis}

In this section we perform a detailed kinematical analysis of the $\mathrm{N}$ and S sources, trying to assess whether they show the disturbances typical of a major-merger system, or are regularly rotating disks.

The QSO and the SMG probably identify two massive galaxies that undergo an interaction or are in the early stages of a major-merger It is therefore possible to estimate the total mass of the system using a simple virial relation such as

$M_{\mathrm{tot}}=d \Delta V^{2} / G=3.2 \times 10^{11} M_{\odot}$,

where $d$ is the observed projected distance $(d=23.5 \mathrm{kpc})$ and $\Delta V$ is the relative velocity $\left(\Delta V=240 \mathrm{~km} \mathrm{~s}^{-1}\right)$. The dynamical mass was estimated assuming that the binary system is observed edge-on and that the galaxies are moving along the line of sight. This estimate is therefore a lower limit for the mass contained within the volume delimited by the location of two sources; obviously, this is also the lower limit of the mass of the dark matter halo hosting the system.

The extended [CII] emission detected in the two sources (Fig. 2) makes a spatially resolved kinematical analysis, which is now described for each source separately. Following Salomé et al. (2012), we first analyzed the position-velocity (PV) diagrams of the $[\mathrm{CII}]$ line and performed a full modeling of the observed kinematics.

\subsection{The submillimeter galaxy BR 1202 North}

The left panel of Fig. 4 shows the [CII] position-velocity diagram for the SMG. It has been obtained by extracting the 

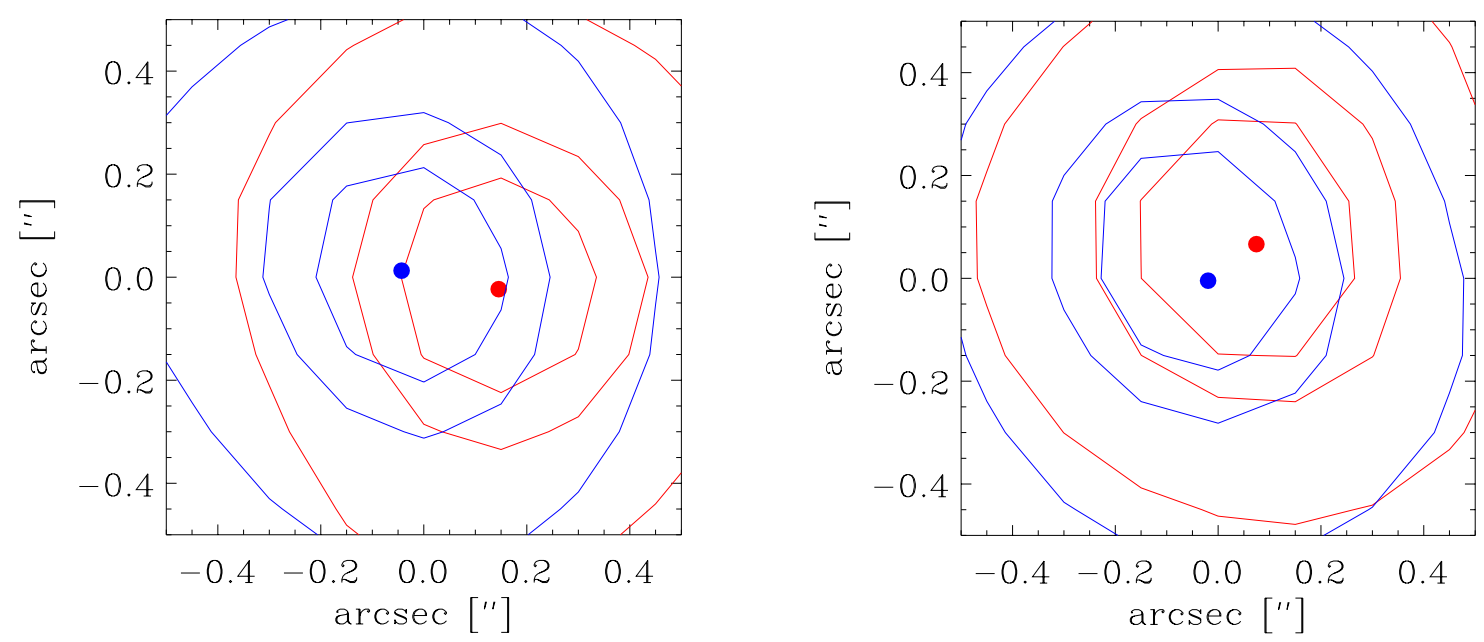

Fig. 5. Surface brightness contours obtained by collapsing the red and blue channels in the SMG (N source, left panel) and QSO (S source, right panel). Contours are at $90 \%, 80 \%$, and $50 \%$ of the peak values.

spectrum from a three-pixel-wide $\left(0.45^{\prime \prime}\right)$ slit centered on the [CII] surface brightness peak and oriented E-W, as in Salomé et al. (2012). The absolute velocity scale is referred to $z=$ 4.6949. This diagram presents at least three main [CII] components at $\sim-350 \mathrm{~km} \mathrm{~s}^{-1}, \sim-280 \mathrm{~km} \mathrm{~s}^{-1}$, and $\sim 250 \mathrm{~km} \mathrm{~s}^{-1}$. As suggested by Salomé et al. (2012) based on the CO PV diagram, the component at about $\sim 250 \mathrm{~km} \mathrm{~s}^{-1}$ probably indicates another weak galaxy east of the SMG, which might be undergoing an interaction or a merger. This component is responsible for the asymmetric line profile of the SMG compared with that of the QSO (Fig. 3). The two main components are separated by $\Delta v=70 \mathrm{~km} \mathrm{~s}^{-1}$, resulting in a double-peaked line profile which, in some cases, can be the signature of a rotating disk. These separate peaks are not seen in the CO PV diagram by Salomé et al. (2012).

Before performing a spatially resolved kinematical analysis, we tested whether the kinematics is indeed spatially resolved and consistent with a rotating disk.

The first test was to perform a spectroastrometric analysis by collapsing all the velocity channels blueward of the line peak and the redward ones in two separate images. If the SMG has a rotating disk and the rotation is resolved, the blue and red channel maps should have offset surface brightness centroids since they map the approaching and receding halves of the disk, respectively. This is indeed shown in the left panel of Fig. 5 where the surface brightness peaks of the blue and red maps are separated by $\sim 0.2^{\prime \prime}$ (1.3 pixels corresponding to $1.2 \mathrm{kpc}$ ) along the E-W direction. Following (Gnerucci et al. 2011b), we can therefore use spectroastrometry to obtain a first estimate of the dynamical mass of the SMG as

$M_{\mathrm{dyn}} \sin ^{2} i=(2.3 \pm 0.2) \times 10^{9} M_{\odot}\left(\frac{F W H M}{100 \mathrm{~km} \mathrm{~s}^{-1}}\right)^{2}\left(\frac{r}{1 \mathrm{kpc}}\right)$

where $i$ is the disk inclination with respect to the line of sight, FWHM is the full width at half maximum of the [CII] emission line emission, and $r$ is the half distance between the "red" and "blue" luminosity centroids. On top of the measurement errors, one should consider a $10 \%$ systematic rms error, deriving from the calibration of Gnerucci et al. (2011b), which was based on a sample of $z \sim 2-3$ Lyman-break galaxies with spatially resolved kinematics. Using $F W H M=500 \pm 40 \mathrm{~km} \mathrm{~s}^{-1}$, $r=0.6 \pm 0.2 \mathrm{kpc}$, the spectroastrometric mass estimate is then $M_{\mathrm{dyn}} \sin ^{2} i=(3.5 \pm 0.4) \times 10^{10} M_{\odot}$, with a systematic error of
$10 \%$ rms. We stress that this value is valid only under the assumption of a virialized rotating disk. Indeed, the results from the spectroastrometric analysis are consistent with the expectations from a virialized rotating disk, but it could also be possible to reproduce them with outflowing gas in a particular configuration and therefore the more accurate analysis described below is required.

The second test consists of analyzing velocity residual map obtained with a pixel-by-pixel kinematical fitting after assuming that the sources are spatially unresolved. In this case, the line profile of the integrated [CII] emission plotted in Fig. 2 is expected to be equal to any line profile regardless of the spatial pixel from where they have been extracted. We therefore performed a pixel-by-pixel fit of the emission line with two Gaussian functions, only allowing for a variation of the total line flux, as expected from unresolved emission. We then obtained the residuals maps by collapsing the blue and red spectral channels, as we did for spectroastrometry. The results of this exercise are shown in Fig. 6, where we show the blue and red residuals (left and right panels, respectively) with the contours of the total line surface brightness superimposed. The structure of the residuals is clearly what we would expect for a rotating disk after a line fit that does not allow for a variation of the line average velocity. Indeed, the residual map from the blue channels shows positive residuals on the left side and negative residuals on the right side, while the opposite takes place in the residual map from the red channels. Therefore the line velocity is bluer than the average one on the left side, and redder on the right side, as already suggested by the spectroastrometry. This is expected for a rotating disk with the line of nodes oriented E-W with the eastern half approaching the observer.

The observed radial profile of the [CII] surface brightness and the two tests performed above strongly suggest that the [CII] kinematics has been spatially resolved. When extracting the line kinematics we took into account that the [CII] line profile is the result of the combined emission of the SMG and of its faint companion observed in the PV diagram at a velocity of $\sim 250 \mathrm{~km} \mathrm{~s}^{-1}$. Since the emission of the companion is probably too weak to resolve its kinematics, we performed a pixel-by-pixel fit with the two Gaussian components, but allowing only the stronger one to vary its average velocity and velocity dispersion. Therefore we obtained the velocity field only for the SMG galaxy, cleaning out the distortions due to the faint component. The velocity and flux 

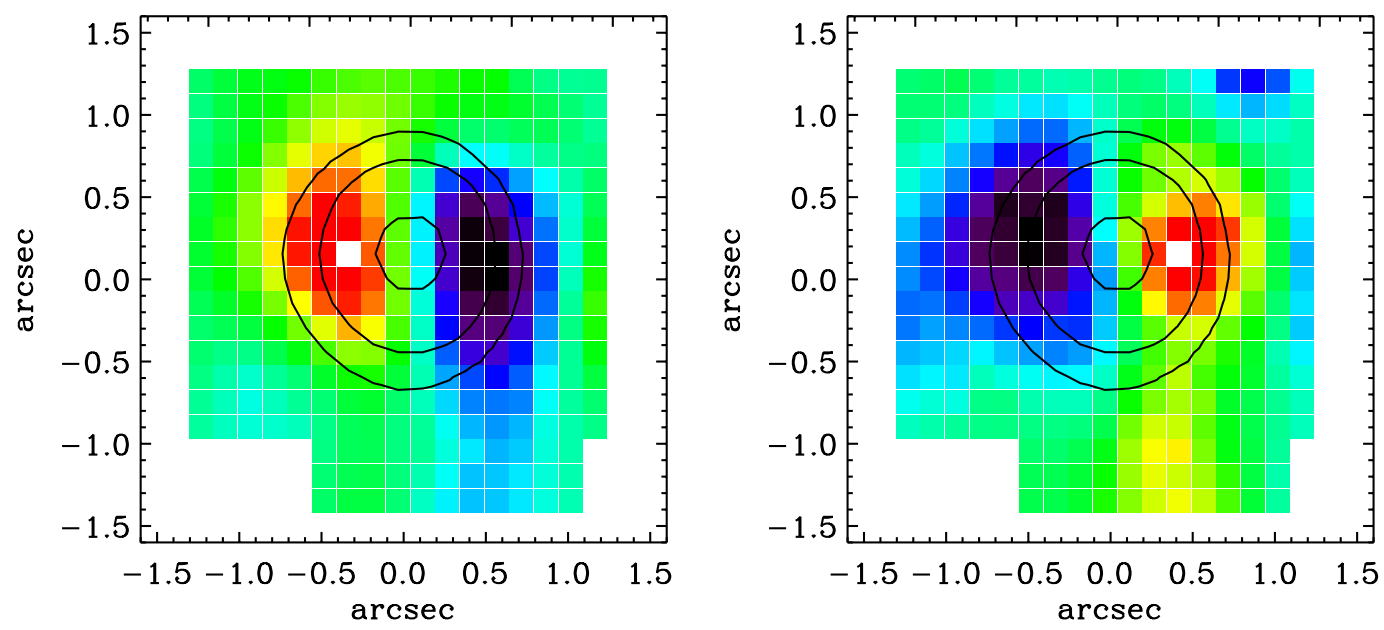

Fig. 6. Blue and red residual maps of the SMG obtained assuming spatially unresolved kinematics (left and right panels, respectively). Contours represent the total line surface brightness at $90 \%, 50 \%$, and $30 \%$ of the peak value.

maps, shown in Fig. 7, were obtained after selecting only those spatial pixels for which the signal-to-noise ratio of the emission line is equal to or higher than 3 ; we defined the signal-to-noise ratio of the emission line as the ratio between the peak of the main Gaussian component and the rms of the residuals.

We then fit the velocity map of the SMG using the kinematical disk model used for $z \sim 3$ Lyman-break galaxies by Gnerucci et al. (2011a). We assumed that the gas is circularly rotating in a thin disk, that the gravitational potential depends only on disk mass, and that the disk surface mass density distribution is exponential $\Sigma(r)=\Sigma_{0} \mathrm{e}^{-r / r_{0}}$, where $r$ is the distance from the disk center and $r_{0}$ is the scale radius. Following Cresci et al. (2009) and Gnerucci et al. (2011a), we also assumed that the mass density distribution is traced by gas emission, and therefore that the center of rotation and scale radius are set by the peak and scale radius of the [CII] surface brightness distribution parameterized by $I(r)=I_{0} \mathrm{e}^{-r / r_{0}}$ (Cresci et al. 2009). Rotation center and $r_{0}$ were then derived by $\chi^{2}$ minimization following the comparison of observed and model surface brightness distributions after convoluting the latter with the spatial PSF of the data. The circular velocity field was projected along the line of sight, weighted by the intrinsic line surface brightness, and then convolved with the spatial resolution of the data to be directly compared with the observed velocity field.

The free parameters of the kinematical model are $i$, the inclination of the disk along the line of sight, $\theta$, the position angle of the disk line of nodes, $V_{\text {sys }}$, the systemic velocity of the galaxy, and $M_{\text {dyn }}$, the total dynamical mass of the disk. $M_{\text {dyn }}$ is actually the disk mass enclosed within a radius of $5 \mathrm{kpc}$. Since this radius is much larger than the disk scale radius, this corresponds to the total mass of the disk. The value of $5 \mathrm{kpc}$ was chosen because the proper distance at $z \sim 5$ corresponds to a proper distance of $10 \mathrm{kpc}$ at $z \sim 2$, which is the value used in the work of Cresci et al. (2009).

The best-fit parameter values for the kinematical model were found using the MPFIT (Markwardt 2009) and AMOEBA algorithms in the IDL environment. The $\chi^{2}$ space was explored using Monte Carlo Markov chains (MCMC), which allow one to sample a target density distribution, or, in other words, allow one to estimate the posterior probability distribution for the $i$ dimensional parameter space that defines our disk models. We used the IDL routines developed for the EXOFAST IDL package (Eastman et al. 2013).
Since the disk inclination is not well constrained from the data and since the dynamical mass could be larger than the molecular gas mass, we considered two cases, one with a totally free $M_{\text {dyn }}$ and the other with a prior on $M_{\text {dyn }}$ based on the molecular gas mass. The prior is given by a lognormal Gaussian step function, that is a lognormal Gaussian on the left side of the peak and a constant value on the right. The peak of the Gaussian is given by the molecular gas mass presented in Table 1, and the dispersion is $0.3 \mathrm{dex}$, corresponding to a factor 2 and compatible with the uncertainties on the conversion factor (e.g. Bolatto et al. 2013).

Figure 7 shows the results of the kinematical model fitting and Table 1 reports the best-fit parameters. Figure 8 shows the confidence contours for the $M_{\text {dyn }}$ and inclination $i$ values obtained from the MCMC with $10^{5}$ trials with (red) and without (green) the dynamical mass prior.

The scale radius here is $r_{0}=2.8 \mathrm{kpc}$, similar to the maximum distance with measured velocity from the disk center, indicating that the observed velocity field traces only the inner regions of the galaxy where dark matter is very likely negligible. It also shows that deviations from the exponential mass density profile due to dark matter are very likely negligible and that $M_{\text {dyn }}$ is indeed close to the total disk mass because $2 r_{0} \sim 5 \mathrm{kpc}$.

It is clear from Fig. 8 that without the prior the best fitting dynamical mass would be smaller than the gas molecular mass by $\sim 0.5$ dex. However, the velocity map of the SMG is limited to the central parts of the disks and, given the spatial resolution, it lacks a clearly defined spider diagram: only the central velocity gradient is well detected. Therefore, the inclination cannot be well constrained from the data: given the well-known degeneracy of mass and inclination, the mass is poorly constrained, too. The use of the prior therefore provides a physically motivated lower limit to the dynamical mass, which helps constraining its value.

We also performed a fit without the proxy on mass and in which the disk inclination was constrained by assuming that the [CII] flux distribution was radially symmetric on the disk: the resulting disk inclination is in the range $i=6^{\circ} \pm 3^{\circ}$ (90\% confidence), which additionally supports the low disk inclination.

To verify whether the rotating disk is really rotationally supported, we compared the intrinsic velocity dispersion with the rotational velocity. By intrinsic velocity dispersion we mean the one that is not accounted for by unresolved rotation and which 

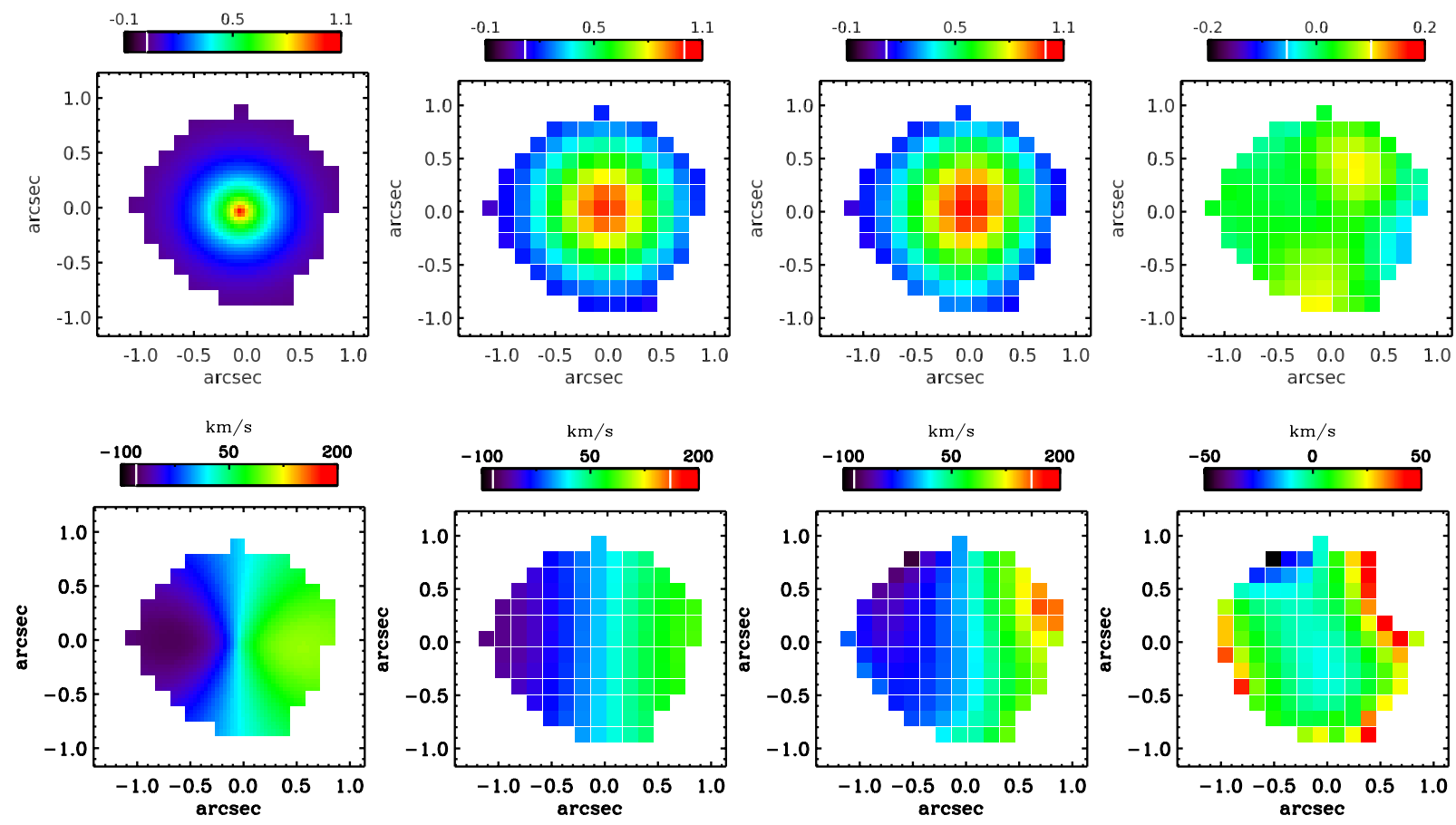

Fig. 7. Analysis of the SMG surface brightness and kinematics. Upper panels: surface brightness fitting with an exponential disk. Lower panels: velocity field fitting with a circularly rotating disk. From right to left: model, model convoluted for PSF, data and residual map.

might be due to turbulent motions. From the dynamical modeling we can estimate the maximum rotational velocity $\left(V_{\max }\right)$ and the intrinsic gas velocity dispersion $\sigma_{\text {int }}$

$\left\langle\sigma_{\text {int }}^{2}\right\rangle=\left\langle\sigma_{\text {obs }}^{2}-\sigma_{\text {model }}^{2}\right\rangle$

where $\sigma_{\text {obs }}$ is the observed velocity dispersion and $\sigma_{\text {model }}$ is that expected from the model that best fits the velocity map. $\sigma_{\mathrm{obs}}$ is directly obtained from the main Gaussian line component without any correction for the spectral resolution; $\sigma_{\text {model }}$ is computed by assuming a cold rotating disk, that is with no intrinsic velocity dispersion, and by taking into account the spectral and spatial resolutions of the observations. In particular, the main contribution to $\sigma_{\text {model }}$ is given by beam smearing, while the effect of the spectral resolution is negligible since line widths are large. $\sigma_{\text {int }}$ is therefore the intrinsic velocity dispersion of the emitting material.

The ratio $V_{\max } / \sigma_{\text {int }}$ was used to indicate whether the kinematic of the galaxy is rotation dominated $\left(V_{\max }>\sigma_{\text {int }}\right)$ or dispersion dominated $\left(V_{\max }<\sigma_{\text {int }}\right)$. For the SMG we obtained $V_{\text {max }} / \sigma_{\text {int }} \sim 1.3$ with $V_{\max } \sim 270 \mathrm{~km} \mathrm{~s}^{-1}$ and $\left\langle\sigma_{\text {int }}\right\rangle \sim$ $200 \mathrm{~km} \mathrm{~s}^{-1}$. This indicates that the SMG has a dynamically hot disk compared with local galaxies for which $V_{\max } / \sigma_{\text {int }} \sim 10$, but comparable with LBGs at redshift $z \sim 2-3$ (Cresci et al. 2009; Gnerucci et al. 2011a).

Summarizing, the mass and inclination of the SMG disk were derived from the posterior distributions of the parameters by considering the median values and the 5 and 95 percentiles and result in $\log _{10}\left(M_{\mathrm{SMG}} / M_{\odot}\right)=10.8 \pm 0.6\left(M_{\mathrm{SMG}}=\right.$ $\left.6_{-5}^{+18} \times 10^{10} M_{\odot}\right)$ and $i=25^{\circ} \pm 15^{\circ}$.

The spectroastrometric estimate performed at the beginning of this section $M_{\mathrm{SMG}, \mathrm{vir}}=(4.7 \pm 1.5) \times 10^{10} M_{\odot}($ for an average inclination value of $i=60 \mathrm{deg}$, taking into account the $0.15 \mathrm{dex}$ systematic error) is perfectly consistent with the dynamical mass measured from the spatially resolved kinematics, confirming the reliability of the spectroastrometric method of Gnerucci et al. (2011b).

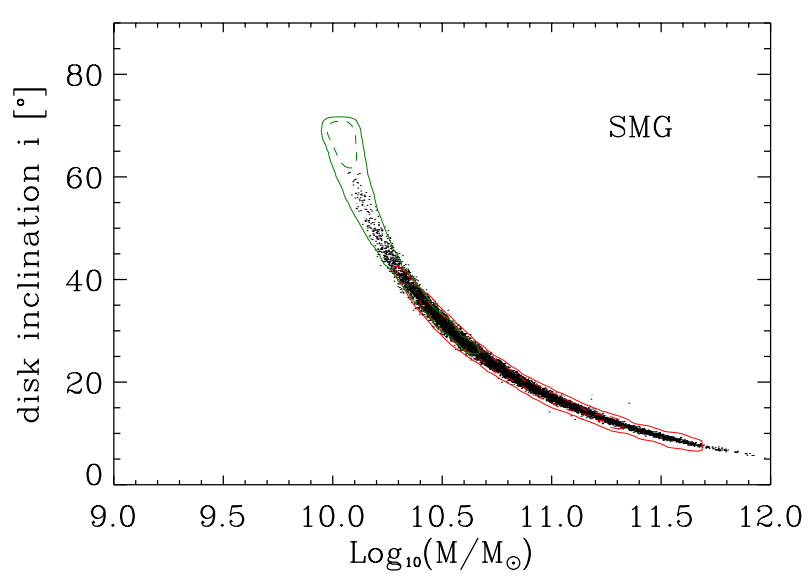

Fig. 8. Confidence contours for the dynamical mass and inclination values of the SMG obtained with the MCMC and $10^{5}$ trials. The solid and dashed lines identify the $95 \%$ and $60 \%$ confidence limits, respectively. The red and green contours denote the cases with and without the prior on the dynamical mass, respectively.

We conclude this section by roughly estimating the amount of mass that could be supported by the disk velocity dispersion, because $M_{\mathrm{SMG}}$ is only the amount of mass that is supported against gravity by disk rotation. If the velocity dispersion indeed provides support against gravity, the dispersion-supported mass would not be negligible since $\sigma_{\text {int }} \sim V_{\text {rot }}$. In this case, unfortunately, it is not possible to apply simple recipes such as the asymmetric drift correction (see, e.g., Barth et al. 2001), and we therefore rely only on a virial estimate. With $\sigma_{\text {int }} \simeq 200 \mathrm{~km} \mathrm{~s}^{-1}$ and $r_{0}=2.8 \mathrm{kpc}$ the dispersion-supported mass is $M_{\mathrm{SMG}, \mathrm{disp}} \simeq$ $\sigma_{\text {int }}^{2} r_{0} / G=2.6 \times 10^{10} M_{\odot}$, similar to the rotation-supported mass, as expected. 

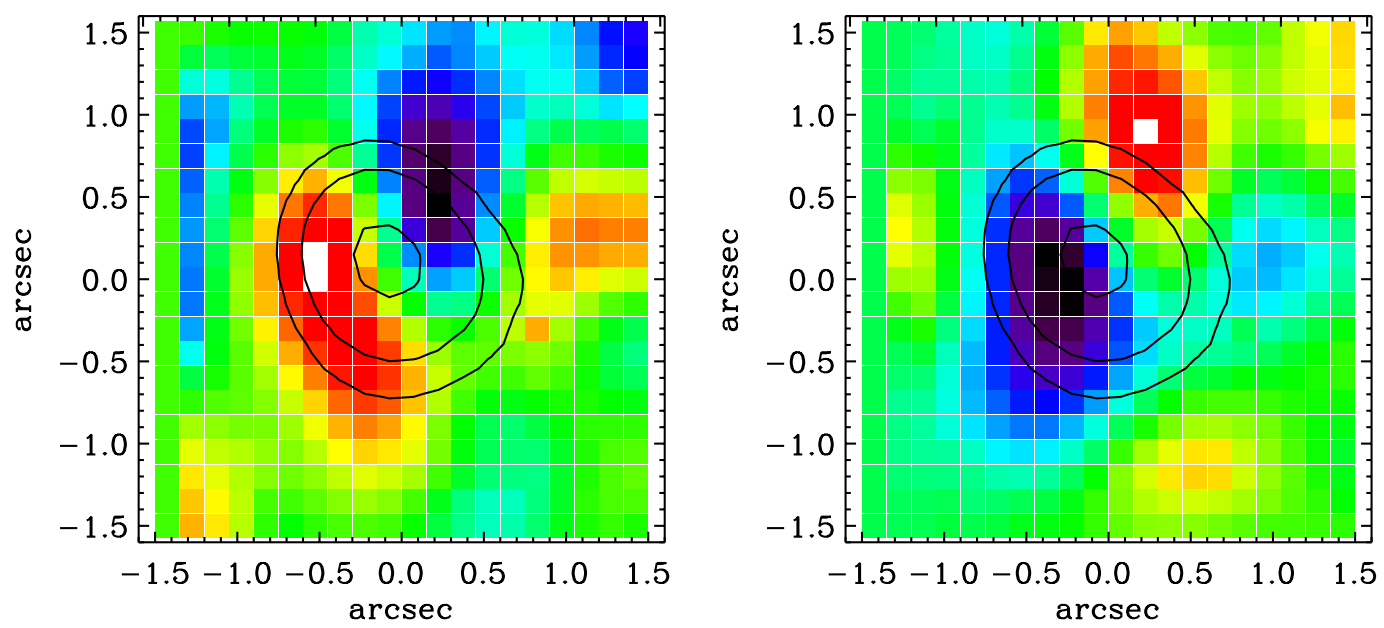

Fig. 9. Blue and red residual maps of the QSO host galaxy obtained assuming spatially unresolved kinematics (left and right panels, respectively). Contours represent the total line surface brightness at $90 \%, 50 \%$, and $30 \%$ of the peak value.
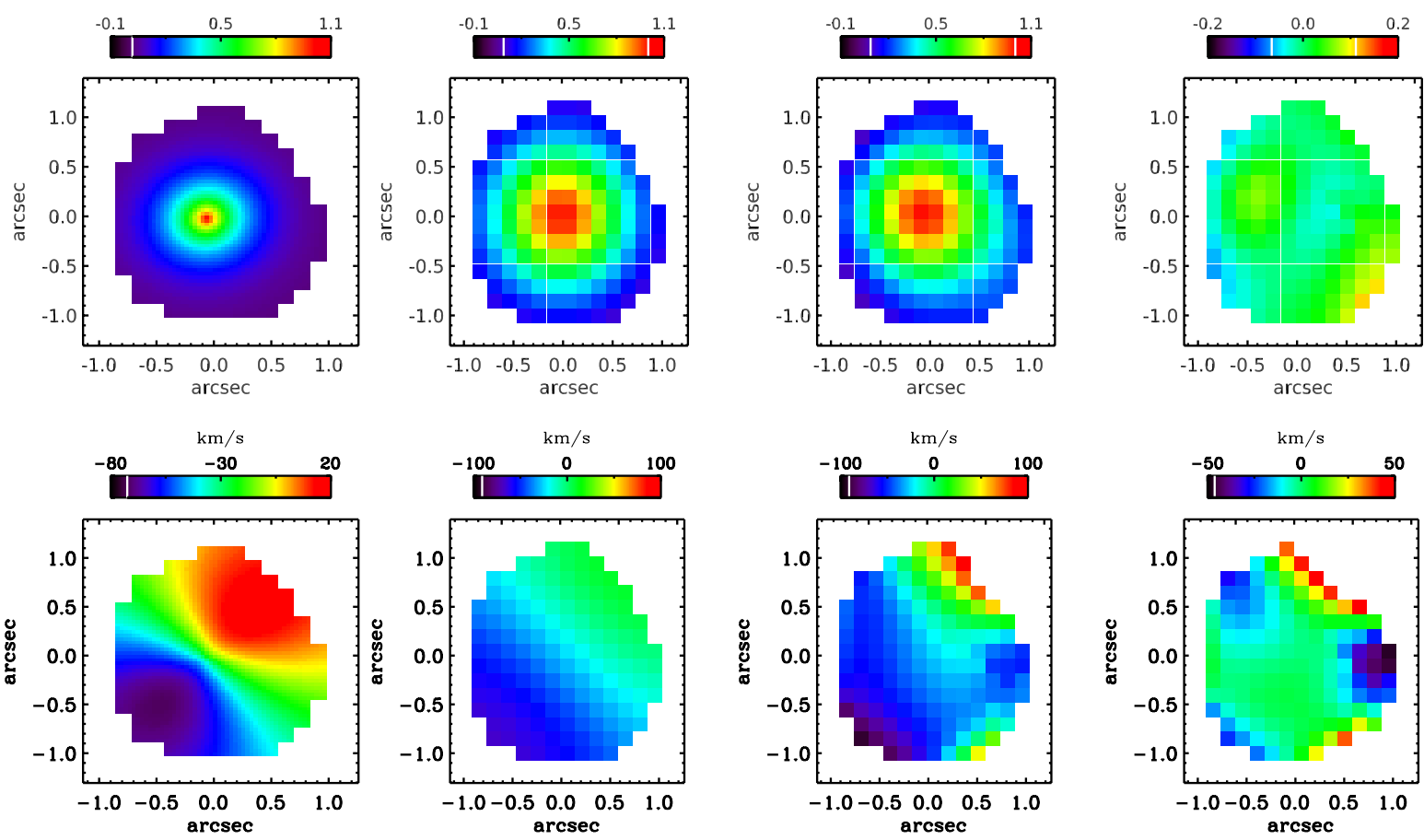

Fig. 10. Analysis of the QSO surface brightness and kinematics. Upper panels: surface brightness fitting with an exponential disk. Lower panels: velocity field fitting with a circularly rotating disk. From right to left: model, model convoluted for PSF, data and residual map.

\subsection{The quasar BR 1202 South}

The [CII] position-velocity diagram for the QSO (Fig. 4, right panel) was obtained by extracting a spectrum from a three-pixelwide $\left(0.45^{\prime \prime}\right)$ slit centered on the surface brightness peak and oriented N-S to compare with Salomé et al. (2012). Similarly to what was found in the PV diagram of the $\mathrm{CO}(5-4)$ line by Salomé et al. (2012), the two main components at $v=-30$ and $40 \mathrm{~km} \mathrm{~s}^{-1}$ a rotating gas disk.

The results of the spectroastrometric test, performed in the same way as for the SMG, are shown in Fig. 5 and confirm the possibility of a rotating disk. The dynamical mass estimated from spectroastrometry following Gnerucci et al. (2011a) is $M_{\mathrm{dyn}} \sin ^{2} i=(8.3 \pm 1.0) \times 10^{9} M_{\odot}$ for measured values of $F W H M=300 \pm 20 \mathrm{~km} \mathrm{~s}^{-1}$ and $r=(0.4 \pm 0.1) \mathrm{kpc}$, the half distance between the blue and red surface brightness centroids.
The results of the residuals tests are shown in Fig. 9 and, as for the SMG, they confirm that the kinematics is spatially resolved and that the disk is probably rotating.

The [CII] kinematical map for the quasar host galaxy has been obtained with a pixel-by-pixel fitting of two Gaussian functions, as suggested by the presence of the red wing shown in Fig. 3. As for the SMG galaxy, we first fit the spectrum obtained from the integrated QSO emission with two Gaussian components, constrained to have the same line width. The fainter component, which reproduces the red wing, was then kept fixed in the pixel-by-pixel fitting except for its flux. The resulting surface brightness and velocity maps are shown in Fig. 10. As for the SMG galaxy, the QSO velocity field shows a velocity gradient oriented in the same direction as the shift between the red and blue centroids. To ensure that the kinematics of the QSO host 
A\&A 559, A29 (2013)

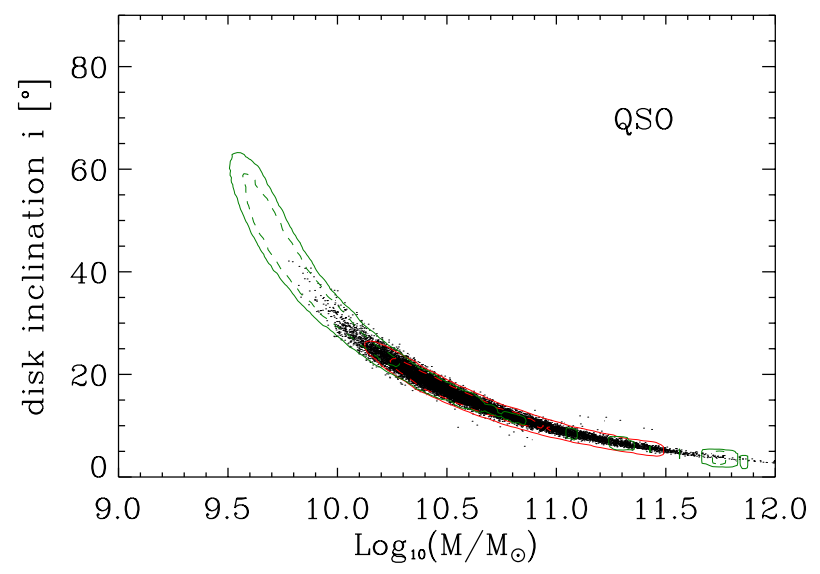

Fig. 11. Confidence contours for the dynamical mass and inclination values of the QSO obtained with the MCMC and $10^{5}$ trials. The solid and dashed lines identify the $95 \%$ and $60 \%$ confidence limits, respectively. The red and green contours denote the cases with and without the prior on the dynamical mass, respectively.

galaxy is not affected by our decomposition method, we furthermore assumed that the red wing was caused by outflows or tidal interactions and therefore is emitted by gas with a high velocity dispersion (Carilli et al. 2013). After constraining the width of the fainter Gaussian function to $\sim 400 \mathrm{~km} \mathrm{~s}^{-1}$ (Carilli et al. 2013), we repeated the previous procedure and obtained a kinematical map that is consistent with the former, within the errors.

We then repeated the analysis performed for the SMG galaxy and fit the QSO kinematics with a rotating disk. We computed an MCMC with $10^{5}$ trials with and without the molecular gas mass prior; the results are shown in Fig. 11, where, as before, the red and green contours denote the cases with and without the prior on the mass. At variance with the case of the SMG, the confidence contours on the two cases overlap at $\log M / M_{\odot} \sim$ 10.6 , indicating that even without the prior, the dynamical mass is consistent with the molecular gas mass. All the relevant bestfit parameter values are also shown in Table 1.

In this case we also performed a fit without the proxy on mass and with the disk inclination constrained by assuming that the $[\mathrm{CII}]$ flux distribution is radially symmetric on the disk: the resulting disk inclination is in the range $i=2^{\circ} \pm 2^{\circ}$ (90\% confidence), which also supports the low disk inclination, as for the SMG.

As in the case of the SMG, by using the rotating disk model we estimated $V_{\text {max }} \sim 230 \mathrm{~km} \mathrm{~s}^{-1}$ and $\left.\mathrm{a}\left\langle\sigma_{\text {int }}\right\rangle\right\rangle \sim 150 \mathrm{~km} \mathrm{~s}^{-1}$ and corresponding ratio $V_{\max } / \sigma_{\text {int }} \sim 1.5$. This indicates a dynamically hot disk compared to local galaxies, but still marginally colder than that of the SMG.

Summarizing, the mass and inclination of the QSO disk derived from the posterior distributions of the parameters by considering the median values and the 5 and 95 percentiles result in $\log _{10}\left(M_{\mathrm{QSO}} / M_{\odot}\right)=10.6_{-0.4}^{+0.8}\left(M_{\mathrm{QSO}}=4_{-3}^{+20} \times 10^{10} M_{\odot}\right)$ and $i=15^{\circ} \pm 10^{\circ}$. This is consistent within the error with the mass estimated from spectroastrometry.

Finally, using $\sigma_{\text {int }} \simeq 150$ and $r_{0}=2.4 \mathrm{kpc}$, the dispersionsupported mass is $M_{\mathrm{QSO} \text {,disp }} \simeq \sigma_{\text {int }}^{2} r_{0} / G=1.2 \times 10^{10} M_{\odot}$, a significant contribution to the rotation-supported mass, as expected.

Overall, the total SMG and QSO mass is smaller than the lower limit we estimated for the dark halo mass.

Intriguingly, the QSO and SMG have similar SFRs (between 1000 and $5000 M_{\odot}$ for both), molecular gas masses $\left(\sim 4 \times 10^{10} M_{\odot}\right)$ and moderately similar dynamical masses when the different inclinations of the gas disks are considered.

\subsection{Spectroscopically detected faint companions}

In Fig. 12 we show the surface brightness maps of the sources identified as faint Gaussian components in the spectra plotted in Fig. 3. These components have velocities of $570 \mathrm{~km} \mathrm{~s}^{-1}$ (SMG) and $500 \mathrm{~km} \mathrm{~s}^{-1}$ (QSO) with respect to the main components, and velocity dispersions of $220 \mathrm{~km} \mathrm{~s}^{-1}$ and $150 \mathrm{~km} \mathrm{~s}^{-1}$. These values where kept fixed during the pixel-by-pixel fit. The surface brightness centroids of these components have a distance of $0.24^{\prime \prime}$ and $0.15^{\prime \prime}$ from the SMG and QSO, respectively, which at the redshift of the BR 1202-0725 system corresponds to $\sim 1.6$ and $\sim 0.9 \mathrm{kpc}$. These components are only detected spectroscopically, and because of their faintness and distance from the main components cannot be seen in direct images obtained by collapsing a given velocity range in the datacube. Therefore these are sources additional to those identified by Wagg et al. (2012) and Carilli et al. (2013).

Figure 13 shows the [CII] channel map for the QSO where, in the channels at velocities from -326 to $-242 \mathrm{~km} \mathrm{~s}^{-1}$, we observe a small weak component whose integrated surface brightness peak is indicated by a cross. For comparison, the filled circle indicates the centroid of the QSO main emission. Its relative velocity with respect to the quasar is $\sim-250 \mathrm{~km} \mathrm{~s}^{-1}$ for a projected distance of $r=3.4 \mathrm{kpc}$.

Overall, we have spectroscopically identified three new components at distances less than $3.5 \mathrm{kpc}$ from the SMG and QSO host galaxy. Considering also the two components identified by Wagg et al. (2012) and Carilli et al. (2013) which correspond to the Ly- $\alpha$ emitters visible in the HST images, we have a total of at least five faint components on top of the two main components, which are the SMG and QSO host galaxy. The BR 1202 system clearly represents a complex system, probably a dense group or protocluster in the early Universe with intense ongoing star formation. However, it is not possible to assess whether these components represent small galaxies, or gas clouds in tidal tails, inflows or outflows. They are probably they are the same type of objects as Ly- $\alpha$ emitters.

However, assuming that they are subjected only to gravitational motions, it is possible to use these components for a rough estimate of the mass of the associated main component (SMG or QSO). Assuming that their orbital planes are seen edge-on and that their motions are entirely along the line of sight, we can combine their velocities and distances from the main components to obtain a virial mass estimate of the SMG or QSO host galaxy. Given our assumptions, these are clearly lower limits on the true mass values. The inferred masses are $\sim 1.2 \times 10^{11} M_{\odot}$ for the SMG and $\sim 5.2 \times 10^{10} M_{\odot}, \sim 5.9 \times 10^{10} M_{\odot}$ from the redshfited and blueshifted components of the QSO. These lower limits should be compared with the measurements performed in the previous sections, which are $0.6_{-0.4}^{+1.9} \times 10^{11} M_{\odot}$ for the SMG and $0.4_{-0.2}^{+2.1} \times 10^{11} M_{\odot}$ for the QSO. These values are evidently agree in remarkably with the measurements from the spatially resolved kinematics supporting the hypothesis of gravitational motions for the spectroscopically detected components. However, it should be noted that (i) any correction for the unknown geometrical projection effects will increase the mass estimate; (ii) the SMG and QSO host galaxy masses enclosed within the measured distances from the faint components are smaller than the total dynamical estimates; and (iii) the above estimates are based on the assumption of circular orbits while, 

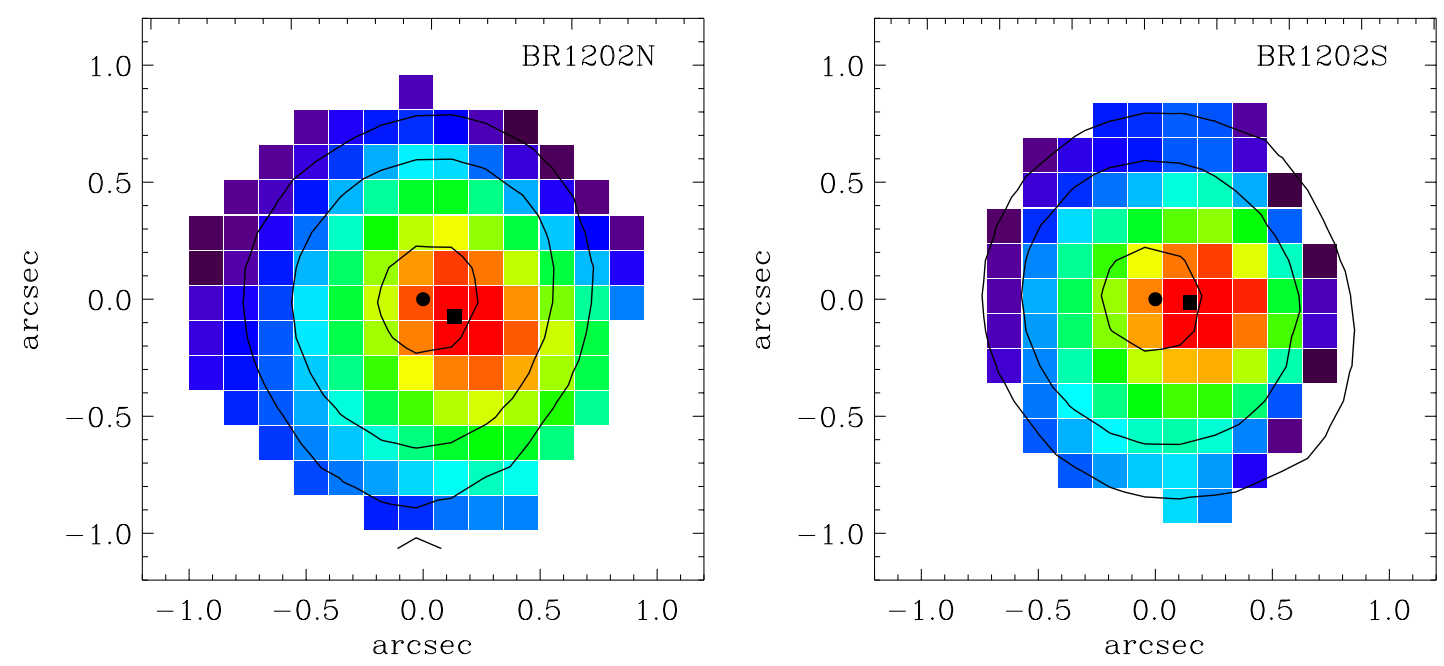

Fig. 12. Surface brightness maps of the sources identified as faint components in the spectral fits: the left and right panels show the SMG and QSO, respectively. The black contours denote the surface brightnesses of the main spectral components; the filled circles and squares denote the centroids of the main and faint components, respectively.

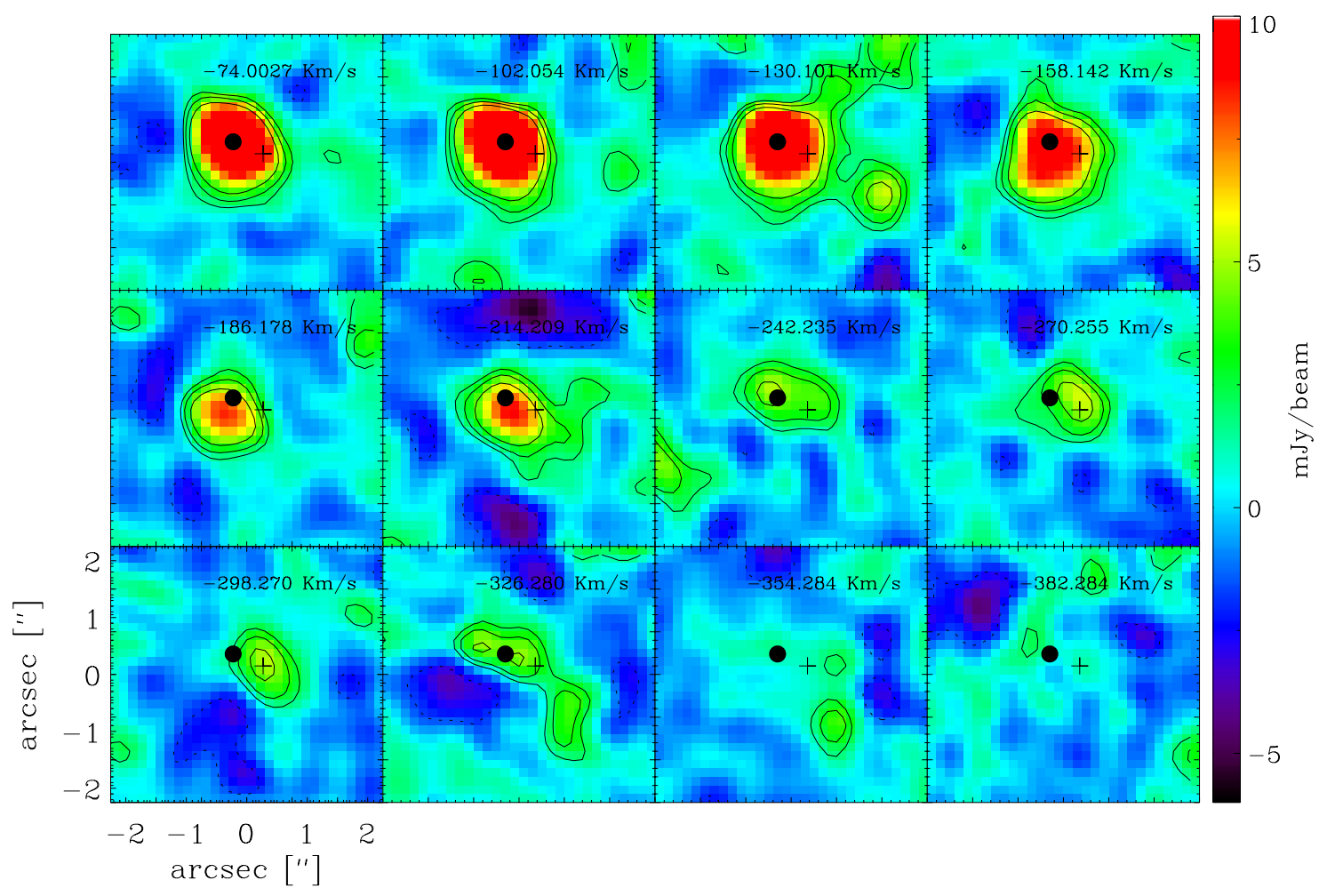

Fig. 13. Brightness per velocity channel in the QSO. Color-coded values and contours at $[2,3,5] \sigma$ levels are shown. The numbers in the upper right corners of the panels denote the central velocity of the channel. The filled circle represents the peak of the integrated surface brightness of the quasar, while the cross represents the peak of the surface brightness of the faint companion, which is detected in channels $\sim-362:-242 \mathrm{~km} \mathrm{~s}^{-1}$.

higher velocities could be found with other, more general, types of orbits. Both (i) and (ii) will undoubtedly worsen the agreement with the dynamical masses at an unknown level; while (iii) would improve it.

It is also possible that these faint components represent quasar- or star formation-driven outflows. This interpretation has been proposed by Carilli et al. (2013) for the red wing of the QSO. However, outflows detected from emission lines at submm wavelengths are seen on both blue and red sides of the emission line profiles (e.g., Feruglio et al. 2010; Maiolino et al. 2012). Moreover, it would be very peculiar that we are seeing almost face-on galactic disks for the SMG and QSO while detecting only outflows on the far sides.

Summarizing, although it is not possible to assess the physical origin of the faint spectral components, the relative velocities and positions of the faint components detected spectroscopically suggest that they are orbiting in the gravitational wells of the SMG and QSO host galaxy, favoring their identification as small galaxies, or gas clouds in an accretion flow or in tidal stream. The relative velocities and positions provide no support either for their identification as gas clouds in QSO or star formation driven outflows. ALMA observations with higher sensitivity and 


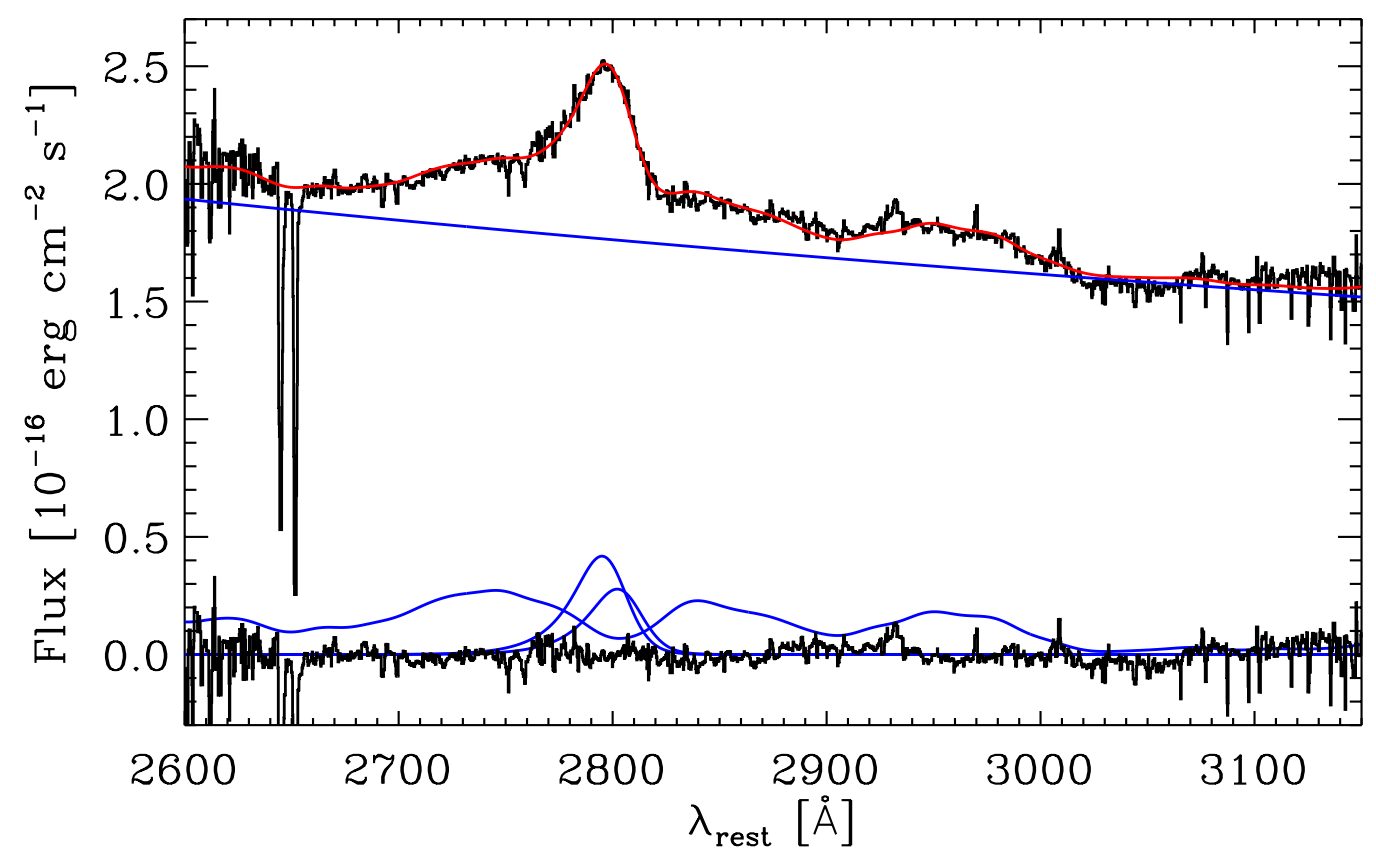

Fig. 14. X-shooter spectrum of the QSO (S source) and model fit (red line) in the good atmospheric regions. The MgII doublet, FeII spectrum and power law continuum are shown with blue lines. Below is the residual spectrum.

spatial resolution are needed to establish the physical origin of these faint spectroscopic components.

\section{Black hole mass of the QSO}

To estimate the black hole mass in the QSO and compare it with the mass of the host galaxy, the only possibility is to use a virial estimate based on broad emission lines such as $\mathrm{H} \beta$, MgII $\lambda 2800 \AA$, and CIV $\lambda 1546 \AA$. H $\beta$ and MgII are currently believed to be the most accurate $\mathrm{BH}$ mass estimators with systematic uncertainties of up to $\sim 0.5 \mathrm{dex}$, while CIV is very likely affected by outflows or nonvirial motions (see Shen 2013, for a review).

An intermediate-resolution spectrum of the QSO has been obtained with X-shooter (Vernet et al. 2011) at the ESO VLT (P.ID. 084.A-0780B) with the main aim of studying galactic and intergalactic absorption lines. A total of 2.5 hours of exposure time was obtained using a slit set of $0.8^{\prime \prime} / 0.7^{\prime \prime} / 0.6^{\prime \prime}$ for the blue, visual (VIS), and near-infrared (NIR) arms, resulting in spectral resolutions of 6000,11000 , and 7950 , respectively. The data were reduced with the public X-shooter pipeline (Modigliani et al. 2010) using the default parameters. The pipeline rebins the spectra to $0.4 \AA /$ pixel in the blue and VIS arm and to $1 \AA /$ pixel in the NIR arm. Telluric absorption was removed using the spectrum of the B5V star Hip 073345 after removing the prominent $\mathrm{H}$ absorption lines. The NIR portion of the spectrum (extending from 1 to 2.5 mum) includes the broad MgII emission line doublet at $2800 \AA$ rest frame, which is considered a good estimator for the mass of the supermassive black hole. The region of the spectrum around the MgII emission is shown in Fig. 14.

The spectrum was fit with a combination of power-law continuum, FeII emission and broad line. For the FeII emission we used a combination of model templates generated with CLOUDY (version 10.00; Ferland et al. 2013) at the conditions typical of the BLR and allowing for Doppler broadening with a Gaussian of given velocity dispersion. For each of the MgII lines we used a broken power-law profile (Nagao et al. 2006) convolved with a Gaussian with unknown velocity dispersion. The two MgII lines were forced to have the same line profile in velocity, with a flux ratio $\lambda 2796 / \lambda 2803$ limited in the $1.0-2.0$ range (Laor et al. 1997). The red line in Fig. 14 shows the best model fit, which results in the following line width and continuum luminosity for the MgII lines: $F W H M=3400 \pm 100 \mathrm{~km} \mathrm{~s}^{-1}$ and $\lambda L_{\lambda}(3000 \AA)=(1.1 \pm 0.1) \times 10^{47} \mathrm{erg} / \mathrm{s}$. Extrapolating the powerlaw continuum, we obtain $\lambda L_{\lambda}(1450 \AA)=1.3 \times 10^{47} \mathrm{erg} / \mathrm{s}$ and $\lambda L_{\lambda}(5100 \AA)=9.5 \times 10^{46} \mathrm{erg} / \mathrm{s}$.

The black hole mass was estimated using the virial relation $M_{\mathrm{BH}}=f V^{2} R / G$, where $V$ is the line width (FWHM or $\sigma)$ and $R$ is the broad line region radius derived from the wellknown radius luminosity relation (Kaspi et al. 2000; Bentz et al. 2008). The scaling factor $f$ was calibrated by imposing that virial masses agree with the relations between $\mathrm{BH}$ mass and host galaxy properties observed in the local Universe (see, e.g., Peterson 2011). Here we adopted the very recent calibration by Marconi et al. (in prep.), obtained by matching the virial BH masses of the AGN with reverberation-mapping data with the $\mathrm{BH}$ masses estimated from the velocity dispersions and luminosities of the host galaxy spheroids, using the $M_{\mathrm{BH}}-\sigma$ and $M_{\mathrm{BH}}-L$ relations by McConnell \& Ma (2013): the adopted virial relation is then $M_{\mathrm{BH}} / M_{\odot}=10^{6.7} F W H M_{1000}^{2}\left[\lambda L_{\lambda}(3000 \AA)\right]_{44}^{0.5}$ where the subscripts 1000 and 44 indicate units of $1000 \mathrm{~km} \mathrm{~s}^{-1}$ and $10^{44} \mathrm{erg} / \mathrm{s}$, respectively. The zero point of this relations has a $\sim 0.3$ dex systematic uncertainty and is lower by $0.15 \mathrm{dex}$ with respect to the zero point of the calibration by Vestergaard \& Osmer (2009). Finally, the estimated black hole mass is $M_{\mathrm{BH}}=1.9 \times 10^{9} M_{\odot}( \pm 0.3 \mathrm{dex}$, systematic $)$. The quasar bolometric luminosity was estimated from the UV and optical luminosities. Using the relation by Mor et al. (2012), we derived $L_{\mathrm{AGN}} \sim 3.8 \times 10^{47} \mathrm{erg} / \mathrm{s}$ from $\lambda L_{\lambda}(1450 \AA)$, while we derived $L_{\mathrm{AGN}} \sim 5.7 \times 10^{47} \mathrm{erg} / \mathrm{s}$ adopting $L_{\mathrm{AGN}} \sim 6 \lambda L_{\lambda}(5100 \AA)$ from Marconi et al. (2004). The Eddington ratio is therefore $L / L_{\text {Edd }} \sim 1.5$. Finally, combining the $\mathrm{BH}$ with the dynamical mass for the host galaxy of $\sim 4 \times 10^{10} M_{\odot}$, the ratio between the black hole and dynamical galaxy mass is $\sim 0.05$; if we also consider the contribution from the velocity dispersion to the host 


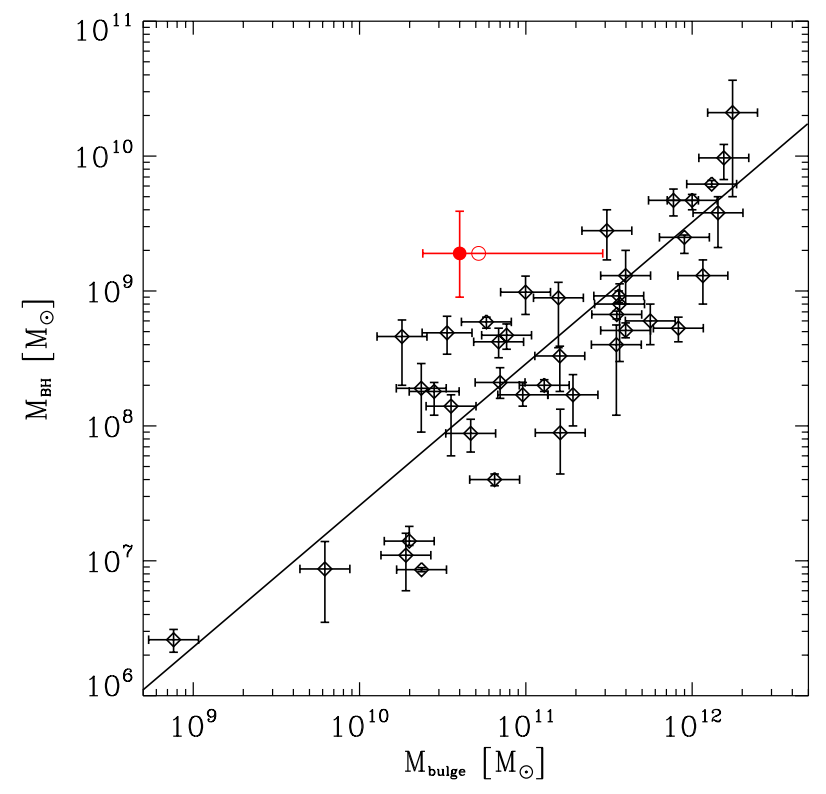

Fig. 15. $M_{\mathrm{BH}}-M_{\text {bulge }}$ relation for 35 early-type galaxies in the local Universe (empty diamonds) and BR 1202 S (red filled circle; the empty filled circle also considers the contribution from the dispersionsupported mass). The black line represents the best-fitting relation estimated by McConnell \& Ma (2013): $\log \left(M_{\mathrm{BH}} / M_{\odot}\right)=8.46+$ $1.05 \log \left(M_{\text {bulge }} / 10^{11} M_{\odot}\right)$.

galaxy mass, this ration drops to 0.04 . These values are consistent with the upper envelope of the local $M_{\mathrm{BH}}-M_{\mathrm{gal}}$ relation (see, e.g., Fig. 3 of Sani et al. 2011 and Fig. 15 above).

\section{Discussion}

According to the paradigm of co-evolution between BHs and their host galaxies, large bursts of star formation and AGN activity should be induced by mergers or interactions, at least at large galaxy masses: gravitational torques cause gas inflows that fuel star formation and accrete onto supermassive black holes in galaxy nuclei. In this framework it is predicted that an SMG and a QSO represent two distinct phases (see, e.g., Alexander \& Hickox 2012, for a recent review). The SMG represents the early phases after a major merging of gas-rich galaxies when star formation and $\mathrm{BH}$ accretion proceed at very high rates in a dustobscured environment. When the AGN is powerful enough, it can expel the gas from the host galaxy, quenching both star formation and black hole accretion, and we are left with a QSO shining in an otherwise passive galaxy (e.g. Di Matteo et al. 2005; Hopkins et al. 2010, 2006).

The BR 1202-0725 system, composed of a SMG (N Source) and of a QSO (S Source) at $z \sim 4.7$, is an ideal test bench for this paradigm. The physical properties of the SMG and of the quasar are summarized in Table 1. Overall, the two galaxies have remarkably similar FIR continuum, [CII] and CO line luminosities, which can be translated into similar SFRs and molecular gas masses. Moreover, they also have similar dynamical masses.

These two galaxies are highly gas rich $\left(M_{\mathrm{mol}} \sim 4 \times 10^{10} M_{\odot}\right)$ and strongly star forming $\left(S F R \sim 3000 M_{\odot} / \mathrm{yr}\right)$ with dynamical masses within the inner $5 \mathrm{kpc}$ of about $M_{\text {dyn }} \sim 5 \times 10^{10} M_{\odot}$ which suggests high gas fractions. Taking into account the uncertainties on the disk inclinations and on the conversion factor, the corresponding gas fractions are constrained in the ranges $0.1-$ 1.0 (0.7 average) for the QSO and $0.2-1$ ( 0.8 average $)$ for the SMG. The average values in parenthesis are for the average disk inclinations of $i=25^{\circ}$ for the SMG and $i=15^{\circ}$ for the QSO. Although weakly constrained, the data suggest moderately high to high gas fraction values.

The relative proximity of the SMG and QSO (projected distance of $\sim 24 \mathrm{kpc}$ ) suggests that the two galaxies might be in the early phases of a major merger that is taking place within a relatively massive halo: the relative velocities of the two galaxies combined with the projected distance allow one to obtain a lower limit to the mass of the host halo, which is $>3.2 \times 10^{11} M_{\odot}$, compared with a total mass of $1.0_{-0.8}^{+3.8} \times 10^{11} M_{\odot}$ for the QSO and SMG.

Summarizing, the SMG and QSO have very similar characteristics, and the only real difference is that the $\mathrm{S}$ source is a QSO, although it is not possible to exclude the presence of a luminous obscured AGN even in the SMG. The intriguing results are that these high SFRs are apparently not triggered by major mergers and that there are no evident signs of feedback in action.

\subsection{Rotating disks and the origin of the high star formation rates}

At variance with the major-merger scenario, the kinematical analysis we have performed indicates that both galaxies have velocity fields that are consistent with regular rotation, without indications of significant distortions like those expected from major mergers, strong interactions, or AGN feedback. For the merger scenario, this fact confirms that the two galaxies are still far enough away from each other not to be affected by significant tidal forces.

Both galaxies are experiencing star formation at rates that will exhaust the gas on very short timescales. The observed molecular gas masses and SFRs imply gas depletion time scales of $\tau_{\text {dep }}=M_{\text {gas }} / S F R \sim 10^{7} \mathrm{yr}$, similar with those of ULIRGs (e.g Tacconi et al. 2006; Bouché et al. 2007). If the SMG and QSO are in an early merger phase, their gas might be entirely converted into stars before they actually do merge. In fact, the relative velocity between the two sources is about $240 \mathrm{~km} \mathrm{~s}^{-1}$ which, combined with a projected distance of $23.5 \mathrm{kpc}$, suggests a merger timescale of $\sim 10^{8} \mathrm{yr}$, longer than the gas depletion timescale. Overall, there are no signs of major-merger induced star formation, and the stars in the galaxies might be already formed when the two galaxies are close enough to merge.

The question that naturally arises is what triggered the burst of star formation in the SMG and the QSO since, apparently, it is not a major merger.

Both the SMG and QSO in the BR 1202-0725 system are experiencing high star formation rates $\left(S F R>1000 M_{\odot} \mathrm{yr}^{-1}\right)$ in rotating disks, apparently excluding major mergers. However, both galaxies show fainter companions that are revealed spectroscopically and whose emission lines have been deblended when measuring the kinematics (see also Salomé et al. 2012). The velocities and positions of these companions relative to the SMG and the QSO are consistent with gravitational motions within the gravitational potential wells of the two largest sources. Furthermore, Carilli et al. (2013) and Wagg et al. (2012) find evidence for the emission of a faint southwest source which is probably interacting with the $\mathrm{S}$ source, and also an additional companion between the two galaxies. Therefore the BR 12020725 system might be a massive proto-cluster where the high star formation activity is probably triggered by minor mergers or interactions that destabilize the gaseous disks, but that do not significantly affect the kinematics of the SMG and QSO galaxies. Alternatively, the high SFR might be triggered by cold gas inflows from the halo, which, again, destabilize the gas disks 
gravitationally. Intriguingly the trigger of star formation from minor mergers, interactions or disk instabilities might be consistent with the dynamically hot disks, indicated by the observed ratios $V_{\max } / \sigma_{\text {int }} \sim 1.3$ and 1.5 for the SMG and for the QSO respectively. Moreover, both velocity maps show residuals at the positions of the possible faint companions after subtracting the rotating disk models. However, the sensitivity and spatial resolution of the data do not allow us to quantify this result.

Evidence that processes such as accretion of pristine gas might induce star formation is starting to accumulate. For instance, Cresci et al. (2010) found evidence that gas accretion might be the origin of metallicity gradients in high-redshift starforming galaxies. In a recent study, Kaviraj et al. (2013) estimated that only $\sim 27$ percent of the total star formation budget in a sample of 80 massive galaxies a $z \simeq 2$ is due to major mergers. This suggests that other contributions to star formation activity such as cold flows and minor merger might be operating. The dynamically hot rotating disks are also found in LBGs at $z \sim 1-2$ (e.g. Genzel et al. 2006; Förster Schreiber et al. 2006) and at $z \sim 3$ (Gnerucci et al. 2011), associated with the very high gas fractions that can destabilize the disk gravitationally (Tacconi et al. 2010; Daddi et al. 2010a). Future observations with ALMA at higher spatial resolution and sensitivity might help to reveal the trigger of the strong star formation activity in BR 1202 and in other systems.

To explore the origin of the star formation activity in more detail, we located the two galaxies on the Schmidt-Kennicutt relation at high redshift. With the disk scale radii estimated during the kinematic analysis, we estimated the SFR and gas surface densities, $\Sigma_{\mathrm{SFR}}$ and $\Sigma_{\text {gas }}$ in the two sources. The values obtained for the $\operatorname{SMG}\left(\Sigma_{\mathrm{SFR}}=80 M_{\odot} / \mathrm{yr} \mathrm{kpc}^{-2}, \Sigma_{\text {gas }}=1900 M_{\odot} \mathrm{pc}^{-2}\right)$ and QSO $\left(\Sigma_{\mathrm{SFR}}=180 M_{\odot} / \mathrm{yr} \mathrm{kpc}^{-2}, \Sigma_{\text {gas }}=1800 M_{\odot} \mathrm{pc}^{-2}\right)$ are perfectly consistent with the relation found by Daddi et al. (2010) for starburst galaxies (SMGs and ULIRGs, see their Fig. 2), which form a different relation from that of local star-forming galaxies.

Summarizing, the lack of major-merger-induced features combined with the high SFR per unit mass suggest that star formation in the BR 1202-0725 system is probably triggered by interactions, minor mergers, or inflow of gas from the halo that increase the concentration of the gas and dust in the galaxy and destabilize the disks gravitationally.

\subsection{Relation between the accreting $\mathrm{BH}$ and its host galaxy}

First of all, we compared the AGN and star formation luminosities in the QSO with the active sources at similar redshifts observed by Mor et al. (2012). Using the $\lambda L_{\lambda}(1450 \AA)$ value estimated from the $\mathrm{X}$-shooter spectrum and combining it with the bolometric correction by Mor et al. (2012), we derive $L_{\mathrm{AGN}} \sim$ $3.8 \times 10^{47} \mathrm{erg} / \mathrm{s}$. When combining this with $L_{\mathrm{SF}} \sim 7 \times 10^{46} \mathrm{erg} / \mathrm{s}$, the QSO is located above the $L_{\mathrm{SF}} \sim L_{\mathrm{AGN}}^{0.7}$ relation observed for lower luminosity sources (Netzer 2009), but close to the five $z \sim 4.8$ sources observed by (Mor et al. 2012): the location above the local $L_{\mathrm{SF}}-L_{\mathrm{AGN}}$ relation might suggests that the feedback mechanism is still not effective enough to reduce star formation activity (see the discussion in Netzer 2009; Mor et al. 2012). Indeed, the regular kinematics observed for the QSO host galaxy suggest that the feedback process might not yet be affecting star formation.

For the SMG, we did not find any evidence for an accreting $\mathrm{BH}$, but the presence of an obscured AGN is supported by radio and X-ray observations. The radio continuum flux density of the
$\mathrm{N}$ source at $1.4 \mathrm{GHz}$ suggests the presence of a highly obscured AGN (Carilli et al. 2002; Iono et al. 2006). This presence is tentatively confirmed by the X-ray emission observed by Chandra at the SMG position (Iono et al. 2006). However, nothing more can be inferred, apart that from here we did not find any evidence for feedback, either.

Figure 15 shows the most up-to-date $M_{\mathrm{BH}}-M_{\text {bulge }}$ relation in the local Universe obtained by McConnell \& Ma (2013). The filled red dot with error bars marks the location of the QSO from the measurements presented above. The empty red circle marks the location of the QSO if the dynamical mass supported by the intrinsic velocity dispersion is taken into account. We recall that the QSO BH mass is a virial estimate, not from spatially resolved kinematical modeling as for the black points in the figure. Moreover, the mass of the QSO host galaxy is not the bulge mass, but the total dynamical mass enclosed within $5 \mathrm{kpc}$.

The present measurement agrees marginally with the upper envelope of the relation, where the uncertainties on the galaxy mass are taken into account. The best-fit relation by McConnell $\& \mathrm{Ma}(2013), \log \left(M_{\mathrm{BH}} / M_{\odot}\right)=8.46+1.05 \log \left(M_{\text {bulge }} / 10^{11} M_{\odot}\right)$, would predict a BH mass of $\sim 1.2 \times 10^{8} M_{\odot}$, which is a factor 10 smaller than observed, consistent with previous estimates for luminous quasars at high redshift (see, e.g., Willott et al. 2013; Wang et al. 2013; Venemans et al. 2012; Merloni et al. 2010; Decarli et al. 2010; Lamastra et al. 2010; Riechers et al. 2009; Peng et al. 2006). However, the uncertainties on the disk inclination of the QSO host galaxy are such that this measurement could be consistent with the upper envelope of the local relation and in particular with the most massive local galaxy like BCGs (McConnell et al. 2012).

This is the first dynamical mass estimate of a QSO host galaxy at high redshift based on the analysis of the spatially resolved kinematics, the only other one is that of a QSO host galaxy at $z \sim 1.3$ (Inskip et al. 2011). All other measurements are either stellar masses based on photometry (Merloni et al. 2010; Decarli et al. 2010; Peng et al. 2006) or dynamical masses based on simple virial estimates (Willott et al. 2013; Wang et al. 2013; Venemans et al. 2012; Riechers et al. 2009). The former are subjected to large uncertainties and selection biases, which might account for the large $M_{\mathrm{BH}} / M_{\mathrm{bul}}$ observed (Lamastra et al. 2010). The latter are subject to large uncertainties on the unknown geometry and kinematics of the host galaxy. In particular, the present work has shown that it is critical to properly take into account the disk inclination: if we were to assume an average inclination of 60 degrees, the dynamical masses so obtained would have been inconsistent with the molecular gas masses.

Therefore, the only possibility to avoid the large uncertainties and selection biases on stellar masses and the large uncertainties on virial mass estimates is to use dynamical host galaxy masses obtained from spatially resolved kinematics. Intriguingly, in the only other case in which the dynamical mass of a QSO host galaxy $(z \sim 1.3)$ has been determined from a spatially resolved kinematical analysis, the BH mass and the galaxy mass agree with the local relation (Inskip et al. 2011). This suggests that dynamical mass determinations from submm spectroscopy such as presented here might be much better probes of the cosmological evolution of the $M_{\mathrm{BH}}-M_{\mathrm{gal}}$ relation.

\section{Conclusions}

We performed a kinematical analysis of the [CII] line emission in the BR 1202-0725 system at $z \sim 4.7$ from ALMA science verification observations. The quasar and the SMG are separated by $\sim 24 \mathrm{kpc}$ and are characterized by very high SFRs, 
larger than $\sim 1000 M_{\odot} \mathrm{yr}^{-1}$. Our kinematical analysis reveals that these galaxies apparently have regularly rotating disks, that are seen almost face-on and that indicate dynamical masses of $6 \times 10^{10} M_{\odot}$, and $4 \times 10^{10} M_{\odot}$ for the SMG and QSO host galaxy, respectively. These disks are hotter than the disks of nearby galaxies, since the maximum rotational velocity is similar to the intrinsic velocity dispersion, but they are very similar to the disks of $z \sim 2-3$ Lyman-break galaxies. If the intrinsic velocity dispersion of the gas disks provides support against gravity, these masses could increase up to $\sim 9 \times 10^{10} M_{\odot}$ and $\sim 5 \times 10^{10} M_{\odot}$. Overall, the SMG and the QSO host galaxy are characterized by very similar physical properties in terms of SFRs, molecular gas mass, and dynamical mass.

The existence of these rotating disks suggest the the high star formation and black hole accretion rates are not induced by a major-merger event, at variance with the commonly accepted scenario for very massive galaxies and very high SFRs. We also detected faint components which, after a spectral deblending, were spatially resolved from the main QSO and SMG emissions. The relative velocities and positions of these components are consistent with orbital motions within the gravitational potentials generated by the QSO host galaxy and SMG, suggesting that they are smaller galaxies in interaction or gas clouds in accretion flows of tidal streams. Moreover, we did not find any clear spectral evidence for outflows caused by AGN or stellar feedback.

The rotating disks and the spectroscopically detected faint components suggest that the high SFRs might be induced by interactions or minor mergers with these companions which, however, do not affect the large-scale kinematics of the disks. Alternatively, the strong star formation may be fueled by the accretion of pristine gas from the host halo. Both these processes could explain the relative high intrinsic velocity dispersion.

Finally, the ratio between the black hole mass of the QSO, obtained from new X-shooter spectroscopy, and the dynamical mass of the host galaxy might be comparable with the similarly high value found in very massive local galaxies, suggesting that the evolution of black hole galaxy relations is probably better studied with dynamical than with stellar host galaxy masses.

New ALMA observations with higher sensitivity and spatial resolution are required to confirm the findings presented here.

Acknowledgements. We thank the anonymous referee for a very quick and thoughtful report. We thank Leslie Hunt, Sperello di Serego Alighieri, and Claudia Cicone for comments and discussions. This paper makes use of the following ALMA data: ADS/JAO.ALMA\#2011.0.00002.S is a partnership of ESO (representing its member states), NSF (USA) and NINS (Japan), together with NRC (Canada) and NSC and ASIAA (Taiwan), in cooperation with the Republic of Chile. The Joint ALMA Observatory is operated by ESO, AUI/NRAO and NAOJ. Also based on observations collected at the European Southern Observatory Very Large Telescope, Cerro Paranal, Chile - Programs 084.A-0780(B), P.I. P. Molaro. Support for this publication was provided by the Italian National Institute for Astrophysics (INAF) through PRIN-INAF 2011 "Black hole growth and AGN feedback through the cosmic time" and by the Italian ministry for school, university and reasearch (MIUR) through PRINMIUR 2010-2011 "The dark Universe and the cosmic evolution of baryons: from current surveys to Euclid".

\section{References}

Alexander, D. M., \& Hickox, R. C. 2012, New Astron. Rev., 56, 93 Barth, A. J., Sarzi, M., Rix, H.-W., et al. 2001, ApJ, 555, 685 Beelen, A., Cox, P., Benford, D. J., et al. 2006, ApJ, 642, 694 Benford, D. J., Cox, P., Omont, A., Phillips, T. G., \& McMahon, R. G. 1999, ApJ, 518, L65
Bentz, M. C., Peterson, B. M., Netzer, H., Pogge, R. W., \& Vestergaard, M. 2008, ApJ, submitted [arXiv:0812.2283]

Bolatto, A. D., Wolfire, M., \& Leroy, A. K. 2013, ARA\&A, in press [arXiv: 1301.3498]

Bothwell, M. S., Maiolino, R., Kennicutt, R., et al. 2013, MNRAS, 433, 1425

Bouché, N., Cresci, G., Davies, R., et al. 2007, ApJ, 671, 303

Bouché, N., Dekel, A., Genzel, R., et al. 2010, ApJ, 718, 1001

Carilli, C. L., Kohno, K., Kawabe, R., et al. 2002, AJ, 123, 1838

Carilli, C. L., Riechers, D., Walter, F., et al. 2013, ApJ, 763, 120

Chapman, S. C., Windhorst, R., Odewahn, S., Yan, H., \& Conselice, C. 2003, ApJ, 599, 92

Chapman, S. C., Blain, A. W., Smail, I., \& Ivison, R. J. 2005, ApJ, 622, 772

Chapman, S. C., Ivison, R. J., Roseboom, I. G., et al. 2010, MNRAS, 409, L13

Conselice, C. J., Mortlock, A., Bluck, A. F. L., Grützbauch, R., \& Duncan, K. 2013, MNRAS, 430, 1051

Coppin, K., Chapin, E. L., Mortier, A. M. J., et al. 2006, MNRAS, 372, 1621

Cresci, G., Hicks, E. K. S., Genzel, R., et al. 2009, ApJ, 697, 115

Cresci, G., Mannucci, F., Maiolino, R., et al. 2010, Nature, 467, 811

Daddi, E., Elbaz, D., Walter, F., et al. 2010, ApJ, 714, L118

Dayal, P., Ferrara, A., \& Dunlop, J. S. 2013, MNRAS, 430, 2891

Decarli, R., Falomo, R., Treves, A., et al. 2010, MNRAS, 402, 2453

Dekel, A., Sari, R., \& Ceverino, D. 2009, ApJ, 703, 785

Di Matteo, T., Springel, V., \& Hernquist, L. 2005, Nature, 433, 604

Downes, D., \& Solomon, P. M. 1998, ApJ, 507, 615

Eastman, J., Gaudi, B. S., \& Agol, E. 2013, PASP, 125, 83

Epinat, B., Contini, T., Le Fèvre, O., et al. 2009, A\&A, 504, 789

Ferland, G. J., Porter, R. L., van Hoof, P. A. M., et al. 2013, Rev. Mex. Astron. Astrofis., 49, 1

Ferrarese, L., \& Merritt, D. 2000, ApJ, 539, L9

Feruglio, C., Maiolino, R., Piconcelli, E., et al. 2010, A\&A, 518, L155

Förster Schreiber, N. M., Genzel, R., Bouché, N., et al. 2009, ApJ, 706, 1364

Gebhardt, K., Bender, R., Bower, G., et al. 2000, ApJ, 539, L13

Genel, S., Genzel, R., Bouché, N., et al. 2008, ApJ, 688, 789

Genzel, R., \& Cesarsky, C. J. 2000, ARA\&A, 38, 761

Gnerucci, A., Marconi, A., Cresci, G., et al. 2011a, A\&A, 528, A88

Gnerucci, A., Marconi, A., Cresci, G., et al. 2011b, A\&A, 533, A124

Gültekin, K., Richstone, D. O., Gebhardt, K., et al. 2009, ApJ, 698, 198

Guyon, O., Sanders, D. B., \& Stockton, A. 2006, ApJS, 166, 89

Haarsma, D. B., Partridge, R. B., Windhorst, R. A., \& Richards, E. A. 2000, BAAS, 32, 685

Hainline, L. J., Blain, A. W., Smail, I., et al. 2011, ApJ, 740, 96

Hopkins, A. M., Irwin, M. J., \& Connolly, A. J. 2001, ApJ, 558, L31

Hopkins, P. F., Somerville, R. S., Hernquist, L., et al. 2006, ApJ, 652, 864

Hopkins, P. F., Bundy, K., Hernquist, L., \& Ellis, R. S. 2007, ApJ, 659, 976

Hopkins, P. F., Bundy, K., Croton, D., et al. 2010, ApJ, 715, 202

Hu, E. M., McMahon, R. G., \& Egami, E. 1996, ApJ, 459, L53

Inskip, K. J., Jahnke, K., Rix, H.-W., \& van de Ven, G. 2011, ApJ, 739, 90

Iono, D., Yun, M. S., Elvis, M., et al. 2006, ApJ, 645, L97

Kaspi, S., Smith, P. S., Netzer, H., et al. 2000, ApJ, 533, 631

Kaviraj, S., Cohen, S., Windhorst, R. A., et al. 2013, MNRAS, 429, L40

Kennicutt, Jr., R. C. 1998, ApJ, 498, 541

Kereš, D., Katz, N., Weinberg, D. H., \& Davé, R. 2005, MNRAS, 363, 2

Kovács, A., Chapman, S. C., Dowell, C. D., et al. 2006, ApJ, 650, 592

Lamastra, A., Menci, N., Maiolino, R., Fiore, F., \& Merloni, A. 2010, MNRAS, 405, 29

Laor, A., Jannuzi, B. T., Green, R. F., \& Boroson, T. A. 1997, ApJ, 489, 656

Lilly, S., Le Fèvre, O., Hammer, F., et al. 1996, in New Light on Galaxy Evolution, eds. R. Bender \& R. L. Davies, IAU Symp., 171, 209

Luhman, M. L., Satyapal, S., Fischer, J., et al. 2003, ApJ, 594, 758

Madau, P., Ferguson, H. C., Dickinson, M. E., et al. 1996, MNRAS, 283, 1388

Magnelli, B., Lutz, D., Berta, S., et al. 2010, A\&A, 518, L28

Magnelli, B., Lutz, D., Santini, P., et al. 2012, A\&A, 539, A155

Maiolino, R., Cox, P., Caselli, P., et al. 2005, A\&A, 440, L51

Maiolino, R., Gallerani, S., Neri, R., et al. 2012, MNRAS, 425, L66

Malhotra, S., Kaufman, M. J., Hollenbach, D., et al. 2001, ApJ, 561, 766

Mannucci, F., Cresci, G., Maiolino, R., Marconi, A., \& Gnerucci, A. 2010, MNRAS, 408, 2115

Marconi, A., \& Hunt, L. K. 2003, ApJ, 589, L21

Marconi, A., Risaliti, G., Gilli, R., et al. 2004, MNRAS, 351, 169

Markwardt, C. B. 2009, in Astronomical Data Analysis Software and Systems XVIII, eds. D. A. Bohlender, D. Durand, \& P. Dowler, ASP Conf. Ser., 411, 251

McConnell, N. J., \& Ma, C.-P. 2013, ApJ, 764, 184

McConnell, N. J., Ma, C.-P., Murphy, J. D., et al. 2012, ApJ, 756, 179

Merloni, A., \& Heinz, S. 2008, MNRAS, 388, 1011

Merloni, A., Bongiorno, A., Bolzonella, M., et al. 2010, ApJ, 708, 137

Michałowski, M. J., Dunlop, J. S., Cirasuolo, M., et al. 2012, A\&A, 541, A85 
A\&A 559, A29 (2013)

Modigliani, A., Goldoni, P., Royer, F., et al. 2010, in SPIE Conf. Ser., 7737

Mor, R., Netzer, H., Trakhtenbrot, B., Shemmer, O., \& Lira, P. 2012, ApJ, 749, L25

Nagao, T., Marconi, A., \& Maiolino, R. 2006, A\&A, 447, 157

Narayanan, D., Bothwell, M., \& Davé, R. 2012, MNRAS, 426, 1178

Netzer, H. 2009, MNRAS, 399, 1907

Omont, A., Petitjean, P., Guilloteau, S., et al. 1996, in Molecules in Astrophysics:

Probes \& Processes, ed. E. F. van Dishoeck, IAU Symp., 178, 501

Papadopoulos, P. P., van der Werf, P., Xilouris, E., Isaak, K. G., \& Gao, Y. 2012, ApJ, 751, 10

Peng, C. Y., Impey, C. D., Rix, H.-W., et al. 2006, ApJ, 649, 616

Peterson, B. M. 2011 [arXiv: 1109.4181]

Riechers, D. A., Walter, F., Carilli, C. L., \& Lewis, G. F. 2009, ApJ, 690, 463

Rodighiero, G., Cimatti, A., Gruppioni, C., et al. 2010, A\&A, 518, L25

Salomé, P., Guélin, M., Downes, D., et al. 2012, A\&A, 545, A57

Sani, E., Marconi, A., Hunt, L. K., \& Risaliti, G. 2011, MNRAS, 413, 1479
Sargsyan, L., Lebouteiller, V., Weedman, D., et al. 2012, ApJ, 755, 171

Shankar, F., Crocce, M., Miralda-Escudé, J., Fosalba, P., \& Weinberg, D. H. 2010, ApJ, 718, 231

Shen, Y. 2013, Bull. Astron. Soc. India, 41, 61

Sijacki, D., Springel, V., \& Haehnelt, M. G. 2011, MNRAS, 414, 3656

Tacconi, L. J., Neri, R., Chapman, S. C., et al. 2006, ApJ, 640, 228

Tacconi, L. J., Genzel, R., Neri, R., et al. 2010, Nature, 463, 781

Valiante, R., Schneider, R., Salvadori, S., \& Bianchi, S. 2011, MNRAS, 416, 1916

Venemans, B. P., McMahon, R. G., Walter, F., et al. 2012, ApJ, 751, L25

Vernet, J., Dekker, H., D’Odorico, S., et al. 2011, A\&A, 536, A105

Vestergaard, M., \& Osmer, P. S. 2009, ApJ, 699, 800

Wagg, J., Wiklind, T., Carilli, C. L., et al. 2012, ApJ, 752, L30

Wang, R., Wagg, J., Carilli, C. L., et al. 2013, ApJ, 773, 44

Weiß, A., De Breuck, C., Marrone, D. P., et al. 2013, ApJ, 767, 88

Willott, C. J., Omont, A., \& Bergeron, J. 2013, ApJ, 770, 13 